\title{
ENTRE LA DESPOBLACIÓN Y EL ENVEJECIMIENTO. ESTRUCTURA DEMOGRÁFICA DE UN TEJIDO SOCIAL EN CRISIS. EL CASCO ANTIGUO DE MADRID
}

\author{
POR \\ CARMEN VÁZQUEZ VARELA
}

\section{Crónica de una crisis demográfica anunciada}

Las reminiscencias literarias del título de este primer epígrafe sirven con eficacia a nuestro objetivo, que no es otro que constatar un hecho conocido y largamente debatido en diversos foros en las últimas décadas, el progresivo proceso de despoblación y envejecimiento que padece el distrito Centro madrileño ${ }^{1}$, sin que hasta el momento, y a pesar de las numerosas declaraciones de intenciones, se haya decidido con firmeza instrumentalizar las políticas que hubieran podido contribuir a un progresivo reequilibrio de la estructura demográfica.

Las causas que están en la base de la actual situación son complejas ya que no sólo reflejan los cambios de la estructura poblacional en el ámbito del casco antiguo sino que se imbrican en una evolución de más amplio alcance que afecta, con diferencias regionales, al conjunto de $\mathrm{Eu}$ ropa occidental. En este contexto, la caída de la natalidad y el descenso de la fecundidad, junto con un progresivo incremento de la esperanza de vida al nacer, están en la base del acelerado envejecimiento de los

${ }^{1}$ Fernández Magan C. (1989): «Migraciones centro-periferia en la Comunidad de Madrid: un análisis», Alfoz, 65, pp. 65-70.

Carmen Vázquez Varela: Departamento de Geografía. Universidad de Castilla-La Mancha. Campus de Cuenca.

Estudios Geográficos

Tomo LX, n. ${ }^{\circ} 237$, octubre-diciembre 1999 
efectivos poblacionales de los países de nuestro ámbito ${ }^{2}$, todo ello sin olvidar la ruptura que ha supuesto la progresiva incorporación de la mujer al trabajo así como la influencia del ciclo económico en la demografía. Toda esta serie de condicionantes no ha hecho sino agravar la situación, con especial incidencia en las áreas centrales de las ciudades, donde la carestía de la vida afecta con mayor intensidad al bien vivienda, que, en respuesta a su acelerado encarecimiento y a los cambios acaecidos en la estructura de hogares, disminuye cada vez más su superficie útil. En suma, nos estamos acercando peligrosamente a la imagen de ciudad convertida en refugio para «viejos y ricos» de la que tantas veces hemos oído hablar.

En el caso que aquí nos ocupa, el casco antiguo madrileño, la situación se ha visto agravada por la concurrencia de una serie de factores que ha contribuido a fijar de modo indeleble a una población de bajos recursos y elevada edad, al tiempo que, aquellos otros con mayor capacidad económica salían del distrito ante la progresiva pérdida de calidad residencial y ambiental del entorno ${ }^{3}$. Esta descripción, aunque un poco simplista no por ello menos cierta, se ajusta a grandes rasgos al perfil de la estructura poblacional y sus características socioeconómicas, que sólo en los últimos años ha experimentado pequeñas alteraciones por la llegada de nuevos efectivos, que han contribuido por una parte a un leve rejuvenecimiento de la pirámide de población y por otra a una mayor polarización en la estructura socioeconómica y espacial de sus residentes.

Las perspectivas de población ya parecían claras a finales de los años setenta, cuando, dentro del conjunto de estudios llevados a cabo para el PAI-Centro, se elaboraron una serie de proyecciones que describían factores probables a corto plazo. En el quinquenio inmediatamente anterior a la fecha de elaboración de los cálculos, 1970-1975, el distrito había perdido 37.137 habitantes (el 16\% de su población), lo que ofrecía un saldo migratorio anual de más de 7.400 residentes y, si los ritmos mi-

2 Una recopilación integradora de las aportaciones realizadas a la problemática de las poblaciones envejecidas desde el campo de la geografía se encuentra en: Rowles, G. D. (1986): «The geography of ageing and the aged: toward an integrated perspective», Progress in Human Geography, 4, pp. 511-539.

3 En los procesos de movilidad residencial centro-periferia, también pueden identificarse cambios que tienen como origen el acceso a la vivienda dentro de un régimen de tenencia en propiedad, fenómeno igualmente detectado en diversos países europeos de nuestro entorno: Dieleman, F. M. \& Everaers, P. C. J. (1994): «From renting to owning: life course and housing market circumstances», Housing Studies, 9, 1 pp. 11-25. 
gratorios no se incrementaban, parecía ser debido a que los efectivos absolutos de población, sobre todo en las edades jóvenes y típicamente migrantes, ya estaban enormemente menguados.

Cuadro I

EVOLUCIÓN DE LA POBLACIÓN DEL DISTRITO CENTRO

(1955-1991)

\begin{tabular}{cccc}
\hline \multirow{2}{*}{ Año } & Población de Derecho & \multicolumn{2}{c}{ Índices } \\
\cline { 3 - 4 } & & Base 1955 & Base quinquenio \\
\hline 1955 & 332.973 & 100 & - \\
1960 & 308.009 & 92,5 & 92,5 \\
1965 & 263.840 & 79,2 & 85,7 \\
1970 & 231.408 & 69,5 & 87,7 \\
1975 & 194.271 & 58,3 & 84,0 \\
1981 & 162.571 & 48,8 & 83,6 \\
1986 & 143.677 & 43,1 & 88,3 \\
1991 & 136.125 & 40,8 & 94,7 \\
\hline
\end{tabular}

Fuente: Elaboración propia sobre datos de resúmenes estadísticos del Ayuntamiento de Madrid y Censos de Población.

Como puede verse reflejado en el Cuadro I, el proceso de vaciamiento ha sido de tal calibre que en el breve espacio de 35 años los efectivos poblacionales se han reducido en un $60 \%$, una caída brutal que, sin embargo, no ha mantenido un ritmo uniforme a lo largo de todos lo períodos intercensales. Tras un vaciamiento progresivo desde los años sesenta, las mayores pérdidas, en términos porcentuales, han tenido lugar en la década de los setenta, momento a partir del cual la tendencia se mantiene de forma suavizada, hasta llegar al último período intercensal, 1986-1991, en el que se ha registrado el menor porcentaje de pérdidas desde los años cincuenta, sólo un 5,7\%. Todo parece indicar que, si bien la tendencia no ha llegado a invertirse, al menos se ha conseguido frenar el fenómeno de salida masiva de los residentes tradicionales al tiempo que tímidos procesos de reocupación han comenzado a ponerse en marcha, pero de todo ello hablaremos más adelante.

Por barrios, esta evolución queda bastante más matizada, tal y como puede apreciarse en el Cuadro II, aunque, en este caso, el período con-

$$
-653-
$$




\begin{tabular}{|c|c|c|c|c|c|c|c|c|c|c|c|c|c|}
\hline \multicolumn{14}{|c|}{ CuAdro II } \\
\hline \multicolumn{14}{|c|}{$\begin{array}{c}\text { EVOLUCIÓN DE LA POBLACIÓN POR BARRIOS Y DISTRITO } \\
\text { ENTRE } 1970 \text { Y } 1991\end{array}$} \\
\hline & \multicolumn{13}{|c|}{ Población de derecho } \\
\hline & 1970 & & 1975 & & & 1981 & & & 1986 & & & 1991 & \\
\hline & Total & $\begin{array}{l}\text { Índice } \\
\text { base }\end{array}$ & Total & $\begin{array}{c}\text { Índice } \\
\text { base } \\
1970\end{array}$ & Total & $\begin{array}{c}\text { Índice } \\
\text { base } \\
1970\end{array}$ & $\begin{array}{l}\text { Base } \\
\text { quin- } \\
\text { quenio } \\
\text { ante- } \\
\text { rior }\end{array}$ & Total & $\begin{array}{l}\text { Índice } \\
\text { base } \\
1970\end{array}$ & $\begin{array}{l}\text { Base } \\
\text { quin- } \\
\text { quenio } \\
\text { ante- } \\
\text { rior }\end{array}$ & Total & $\begin{array}{l}\text { Índice } \\
\text { base }\end{array}$ & $\begin{array}{l}\text { Base } \\
\text { quin- } \\
\text { quenio } \\
\text { ante- } \\
\text { rior }\end{array}$ \\
\hline Distrito Centro & 231.408 & 100 & 194.271 & 84,00 & 162.571 & 70,25 & 83,68 & 143.677 & 62,09 & $88,38 \quad 1$ & 136.125 & 58,82 & 94,74 \\
\hline Palacio & 39.179 & 100 & 32.435 & 82,80 & 27.458 & 70,08 & 84,66 & 24.779 & 63,25 & 90,24 & 23.622 & 60,29 & 95,33 \\
\hline Embajadores & 76.537 & 100 & 62.990 & 82,30 & 53.067 & 69,34 & 84,25 & 47.207 & 61,68 & 88,95 & 45.079 & 58,90 & 95,49 \\
\hline Cortes & 19.878 & 100 & 15.965 & 80,30 & 13.541 & 68,12 & 84,82 & 12.238 & 61,57 & 90,38 & 11.271 & 56,70 & 92,10 \\
\hline Justicia & 28.760 & 100 & 24.914 & 86,60 & 20.406 & 70,95 & 61,91 & 16.171 & 63,18 & 89,05 & 16.625 & 57,61 & 91,49 \\
\hline Universidad & 53.295 & 100 & 48.971 & 88,10 & 38.629 & 72,48 & 62,24 & 33.271 & 62,43 & 86,13 & 32.151 & 60,33 & 95,63 \\
\hline Sol & 13.759 & 100 & 10.996 & 79,90 & 9.470 & 68,83 & 86,12 & 8.011 & 58,22 & 84,59 & 7.377 & 53,62 & 92,09 \\
\hline
\end{tabular}

Fuente: Sobre datos de resúmenes estadísticos del Ayuntamiento de Madrid y Censos de Población Elaboración propía.

siderado se limita a las últimas dos décadas. Las tendencias demográficas de los distintos barrios a lo largo de los veinte años precedentes son muy reveladoras. En términos generales, y considerando la evolución a lo largo de todo el período, puede afirmarse que todos los barrios siguen la pauta resumida en el Cuadro I al hablar de la evolución del distrito, es decir, pérdidas de población importantes durante la década de los setenta que van remitiendo paulatinamente al acercarnos a 1991, fecha del último Censo de población realizado. Partiendo de este hecho, un estudio más detallado de los seis barrios por intervalos quinquenales permite aclarar mejor cuáles han sido las tendencias dominantes en cada momento y dentro de cada barrio.

La primera mitad de la década de los setenta resultó de consecuencias nefastas para los barrios de Sol, Cortes, Embajadores y Palacio, que acusaron las pérdidas de población más importantes (especialmente graves en el caso de Sol, que en este quinquenio pierde más de un $20 \%$ de sus efectivos), siempre superiores a la media del distrito, mientras Justicia y Universidad consiguieron retener mayor número de residentes. En la segunda mitad de la década, y en el contexto de crisis demográfica por la que continúa atravesando el distrito, son ahora Justicia y Universidad, que en el quinquenio anterior habían conseguido salir relativamente 
bien librados, los que experimentan descensos superiores a la media; por el contrario, Palacio, Embajadores, Cortes y especialmente Sol consiguen una importante desaceleración en el ritmo de pérdidas. Estos cambios de tendencia inter-barrios constituyen un reflejo de las motivaciones latentes detrás de lo que podríamos denominar la crisis demográfica general. En un primer momento, son los barrios de tradición terciaria, Sol y Cortes, y aquéllos con mayores problemas de adecuación residencial, Embajadores y Palacio, los que acusan las pérdidas de modo más virulento. Sin embargo, en la segunda mitad de la década, son dos barrios como Justicia y Universidad, que mantienen características mixtas con penetración del terciario, especialmente en áreas de borde, e importantes bolsas de infravivienda en sus núcleos interiores de marcado carácter residencial, los que tienen el dudoso honor de encabezar la lista de bajas. Todo parece indicar que las estrategias de vaciamiento poblacional, hasta entonces ligadas a la penetración del terciario en áreas consolidadas y a la expulsión de la población de los barrios tradicionalmente residenciales con serios problemas de vivienda inadecuada, comienzan a diversificar sus cauces de actuación, afectando a barrios cuyas características y posición estratégica dentro del conjunto urbano los hacen susceptibles de acoger nuevas formas de terciarización y recualificación social previa transformación de su tejido residencial. Toda esta serie de transformaciones causará, como más tarde veremos al analizar algunas proyecciones, ciertos desajustes en las previsiones de población realizadas dentro de los estudios del PAI-Centro de 1979.

La década de los ochenta, y dentro de la tónica de paulatina estabilización a la que reiteradamente hemos aludido, se inicia con descensos de población superiores a la media en Sol y Universidad. El freno a la caída continuada de población que, de forma indirecta, supusieron los primeros instrumentos urbanísticos del nuevo Ayuntamiento democrático (Plan Especial de Protección Villa de Madrid, Plan General de Ordenación Urbana, etc.) contribuye a estabilizar a los barrios de marcado contenido residencial y a otros tan fuertemente terciarizados como Cortes; sin embargo, tanto Sol, cuya dinámica de terciarización progresiva parece imparable, como Universidad, sujeto a fuertes presiones especulativas, continúan con una notable dinámica de retroceso.

La segunda mitad de los ochenta permite abrigar ciertas esperanzas sobre las posibilidades de estabilización de la función residencial del distrito. Aunque las tasas siguen siendo negativas, las pérdidas ya no pa- 
recen tan achacables a la salida continuada de población como al propio crecimiento natural de cada barrio, de características fuertemente regresivas dado el marcado envejecimiento de la pirámide de población. En cualquier caso, Justicia, Cortes y Sol se mantienen con pérdidas superiores al conjunto del distrito, lo que nos hace pensar que los instrumentos de control urbanístico no han conseguido frenar el proceso de terciarización tal y como se esperaba, puesto que estos barrios siguen acogiendo nuevos usos en detrimento de su función residencial. Por otra parte, tanto Embajadores como Palacio parecen estar cerca de estabilizar sus efectivos, mientras el barrio de Universidad, que desde mediados de los setenta se había visto afectado por serias tensiones no sólo consigue cuasi estabilizar sus efectivos sino que es ahora el que menos población pierde (poco más de un 3\% en el quinquenio) dentro del conjunto del distrito. A tenor de estos datos, parece posible afirmar que las políticas municipales de rehabilitación y recualificación ambiental llevadas a cabo en el barrio, muy especialmente en el entorno de los PIP (Programas de Intervención Preferente) y más concretamente en la primera experiencia piloto realizada en el área de Conde-Duque, han dado sus frutos y no sólo se ha conseguido frenar el proceso de continua expulsión de la población residente sino que, dada la dinámica inmobiliaria en que ha entrado la zona, también se ha conseguido atraer hacia ella a un número significativo de nuevos residentes cuyas características comentaremos más adelante.

Por último, y antes de pasar a comentar otros datos, nos detendremos brevemente en los resultados de la evolución por barrios en el período considerado. Los índices resultantes, tomando como base el año 1970, nos indican que Sol ha sido con diferencia el barrio más afectado por la pérdida de efectivos poblaciones, reduciendo en veinte años a casi la mitad los que originalmente poseía. A pesar de que en la actualidad su capacidad parece al borde de la saturación y de que las funciones de gestión y dirección tienden a localizarse en ámbitos menos congestionados ${ }^{4}$,

4 Gamir Orueta, A. (1986): «Las sedes sociales de las 500 primeras empresas de España: su implantación en Madrid», (en) Méndez, R. y Molini, F. (Eds.): Descentralización productiva y movilidad industrial en la Comunidad de Madrid, Madrid, Comunidad de Madrid, pp. 125-139; Gamir Orueta, A. (1990): «El terciario decisional en Madrid, Comunidad de Madrid, pp. 125-139; Gamir Orueta, A. (1990): «El terciario decisional en Madrid», (en)Estébanez, J. (Ed.): Madrid, presente y futuro, Madrid, Akal, pp. 150-178, y Gavira, C. (1984): «El espacio del poder (Paseos del Prado, Recoletos y Castellana)», Alfoz, 9, octubre, pp. 39-45. 
su capacidad de atracción, especialmente sobre las funciones comerciales continúa vigente. A cierta distancia, y en un segundo escalón en el ranking de barrios con población recesiva, se sitúan los de Cortes y Justicia. En este caso se trata de dos ámbitos espaciales fuertemente terciarizados, especialmente en el caso de Cortes, que se ven, además, sometidos a las presiones que generan los bordes norte y oeste del distrito (el eje de la Castellana y los conocidos popularmente como «bulevares»). Aunque en su interior todavía quedan importantes núcleos residenciales, éstos han ido cediendo terreno a usos más competitivos que en algunos casos no sólo congestionan el área sino que entran en conflicto directo con los residentes tradicionales que ven su tranquilidad seriamente amenazada por el poder de convocatoria de estos últimos (el caso del área de ocio conocida como «Huertas» en el entorno de la calle del mismo nombre ${ }^{5}$ sería un claro ejemplo de enfrentamiento entre usos residenciales y terciarios debido a la falta de controles sobre licencias de apertura, horarios y niveles de ruido, lo que ha generado graves molestias al vecindario al tiempo que ha incrementado los niveles de inseguridad en la zona).

Y, para acabar, un tercer grupo formado por los barrios de carácter netamente residencial, todos ellos con pérdidas de población inferiores a la media del conjunto. En esta categoría nos encontramos con Embajadores, Palacio y Universidad, los tres con porcentajes de descenso que rondan el $40 \%$. Sin embargo, su comportamiento no ha sido uniforme. Tal y como ya hemos comentado líneas atrás, Palacio y Embajadores, dos barrios con una dinámica relativamente endógena hasta fechas muy recientes, debido a su caracterización socioeconómica y a su particular estructura urbana, pasaron por la fase más aguda de su crisis demográfica en la década de los setenta para ir poco a poco reduciendo las pérdidas y estabilizando sus efectivos poblacionales.

En el caso de Universidad, y aun tratándose de un barrio que conserva un importante volumen de población residente (el segundo tras Embajadores en datos absolutos), difiere de los dos anteriores en la ca-

5 Un estudio realizado para la «zona de Huertas» en 1988 puso de relieve la transformación de usos del suelo en los locales comerciales (plantas bajas) experimentada entre 1977-1987, de donde se había visto desplazado el comercio tradicional por un volumen considerable de locales propios de «ir de copas» (Barral González, L. M.; Pumares Fernandez, P.; Redondo Arandilla, M. y Rodríguez Pose, A. (1989): «El ocio en Huertas: de la bohemia a la modernidad», Alfoz, 61, pp. 83-87). 
dencia de expulsión de la misma. Como ya hemos comentado anteriormente, Universidad acusó la caída de población más importante, en términos relativos, en la década de los ochenta, es decir, con cierto retraso respecto a Palacio y Embajadores. Las razones de esta anomalía son complejas y derivan de las características internas de cada uno de los barrios. En líneas generales, puede afirmase que el origen de las salidas masivas de población en los casos de Palacio y Embajadores ha estado vinculado de modo preferente a la inadecuación de las tipologías residenciales dominantes a los estándares de vivienda que hoy consideramos mínimos. No estamos intentando afirmar que no hayan existido tensiones entre propietarios y residentes a fin de conseguir la evacuación de estos últimos en favor de usos más rentables, pero la falta de presiones, al menos hasta fechas recientes, por parte de actividades terciarias para penetrar en estos barrios induce a pensar que han sido las propias características del stock residencial y del régimen de tenencia, combinadas con las expectativas latentes de los propietarios inmobiliarios, las que de modo preferente han desencadenado el proceso.

El caso de Universidad presenta algunas diferencias, en primer lugar, y aunque conserva en su interior importantes bolsas de infravivienda (barrio de Malasaña), no mantiene las características de uniformidad de los dos ejemplos anteriores. Por otra parte, los fuertes procesos de terciarización iniciados con la apertura del tramo de la Gran Vía y Princesa ${ }^{6}$, y los importantes fenómenos de renovación visibles a lo largo de las calles más prestigiosas del barrio (Bulevares y calle de San Bernardo) han propiciado la competencia espacial entre los usos residenciales tradicionales y los terciarios, frecuentemente encubiertos tras la proliferación de la residencia secundaria, en un espacio mucho más tensionado que los dos anteriores por su posición estratégica dentro de la ciudad. No es sorprendente, por lo tanto, que los momentos más críticos para el barrio se retrasen hasta finales de los setenta-comienzos de los ochenta, momento en que la actividad inmobiliaria recupera el centro como ámbito

6 Al efecto provocado por la apertura de estos dos grandes ejes viarios se sumó en fechas relativamente recientes la total renovación del conocido como barrio de Pozas, en lo que hoy constituye el «triángulo de Princesa», ocupado en buena parte por unos grandes almacenes. Una descripción pormenorizada de toda la operación puede encontrarse en: Álvarez Mora, A. (1979): Las transformaciones del centro-ciudad en el modo de producción capitalista, Madrid, Colegio Oficial de Arquitectos. 
CuAdro III

PROYECCIONES DE POBLACIÓN

\begin{tabular}{|c|c|c|c|c|c|c|c|c|c|c|c|c|c|}
\hline & 1970 & 1975 & $\begin{array}{c}\text { Índice } \\
\text { base }\end{array}$ & $\underset{\ll a »}{1980}$ & Índice & $\begin{array}{c}1980 \\
\ll b »\end{array}$ & Índice & $\begin{array}{c}1980 \\
\ll \mathrm{c} »\end{array}$ & Índice & $\begin{array}{c}1979 \\
\ll d »\end{array}$ & Índice & $\underset{\psi e}{1979}$ & Índ \\
\hline 20 & 32 & 194.271 & 11001 & 193.983 & 99,85 & 161.680 & 83,22 & 167.957 & 88,96 & 220 & 89,16 & 68.198 & 86,58 \\
\hline Palacio & 39.179 & 32.435 & 5100 & 32.431 & 99,99 & 26.797 & 62,62 & 27.891 & 85.99 & 300 & 88,79 & 27.925 & 86,10 \\
\hline Emb & 76.537 & 62.990 & $\begin{array}{ll}0 & 100\end{array}$ & 62.807 & 99,71 & 51.473 & 81,72 & 53.689 & 85,23 & 55.549 & 86,19 & 53.776 & 85,37 \\
\hline Cortes & 19.878 & 15.965 & $5 \quad 100$ & 15.957 & 99,95 & 12.822 & 80,31 & 13.432 & 84,13 & 13.938 & 87,30 & 13.450 & 84,25 \\
\hline Just & 8.760 & 24.914 & 41 & 24.397 & 97,92 & 20.543 & 82,48 & 21.289 & & & 87,96 & 21312 & 85,56 \\
\hline Univ & & & 1100 & 46.921 & & 40. & & & & & 91,75 & 42.177 & 89,79 \\
\hline Sol & 13.759 & 10.271 & 1100 & 11.001 & 100,05 & 8.777 & 79,82 & 9.208 & 83,74 & 9.565 & 86,99 & 9.221 & 83,86 \\
\hline
\end{tabular}

«a» Sin migraciones.

«b» Mismas tasas migratorias.

«c» $80 \%$ de las tasas migratorias del quinquenio 1970-1975.

«» Interrelación lineal edad a edad entre la columna 2 y la 5.

«e» Interrelación lineal entre la columna 2 y la 4.

Fuente: PAI- Centro 1979.

de actuación, toda vez que la crisis de los setenta ha dejado de hacer rentable la construcción en la periferia de grandes conjuntos residenciales. Las posibilidades de rentabilización de una zona como el barrio de Universidad resultarán nefastas para su función residencial original y sólo políticas de recuperación y rehabilitación, como las iniciadas por la EMV (Empresa Municipal de la Vivienda) a partir de 1986, junto con la oferta de nuevos productos residenciales para los sectores más solventes, han conseguido frenar el proceso.

CUADRO IV

PROYECCIONES DE POBLACIÓN PARA EL DISTRITO

\begin{tabular}{lccc}
\hline & Población & $\mathbf{1 9 7 5 = 1 0 0}$ & $\mathbf{1 9 8 0 = 1 0 0}$ \\
\hline 1985-Hipótesis «a» & 165.741 & 85,31 & 98,68 \\
1985-Hipótesis «b» & 158.075 & 81,37 & 94,12 \\
1985-Hipótesis «c» & 143.173 & 73,70 & 85,23 \\
1983-Interpolación & 153.087 & 78,80 & 91,14 \\
1980-1985 (d) & & & \\
\hline
\end{tabular}

«a» Sin migración.

«b» 66\% de las tasas migratorias del quinquenio 1975-1990.

«c» Mismas tasas migratorias que en el quinquenio 1975-1980.

(d) Interrelación lineal edad a edad.

Fuente: PAI-Centro 1979. 
Retomando de nuevo el tema de las proyecciones de población, a las que antes hemos aludido, el contraste entre los datos de los últimos recuentos estadísticos y las previsiones establecidas en las estimaciones del PAI-Centro en 1979 (Cuadros III y IV) ofrece una visión del grado de ajuste de las mismas a la evolución real del distrito. Por otra parte, también hay que señalar, antes de proceder a establecer cualquier comparación entre los datos, que al efectuar las proyecciones se jugó con un cuadro de suposiciones, tomando como punto de partida la población de 1980 que registra la columna 5 del Cuadro III, es decir la calculada en base a: continuidad del comportamiento de la fecundidad y de la mortalidad del período anterior; y, estimación de que las tasas de emigración sufren una reducción homogénea del 20\% respecto al quinquenio anterior. Esta última valoración concreta se justifica por las siguientes razones, muy esquemáticamente resumidas: a) En lo fundamental, los factores de expulsión del Distrito siguen actuando y no ha habido medidas o cambios de estrategias significativas en la actividad de los principales agentes urbanos. $b$ ) Existen varias circunstancias que hacen pensar que la intensidad de este fenómeno va a descender algo, tanto por el descenso de la actividad inmobiliaria de derribo y renovación como por la existencia de un cambio en el cuadro y la actividad administrativa respecto a la construcción (de carácter cautelar esencialmente). c) Los efectivos de población ya son poco numerosos, especialmente en las edades migrantes, por lo que el mantenimiento de un ritmo equivalente de expulsión es difícilmente alcanzable ${ }^{\text {. }}$.

La elección del horizonte citado no parece muy descabellada si comparamos los datos de la columna 5 del Cuadro III con los datos reales que arroja el Censo de 1981 (Cuadro II). En general, y teniendo en cuenta el desajuste que puede representar la diferencia de un año en las previsiones, los barrios de Palacio, Embajadores y Cortes rozan las cifras apuntadas, mientras Justicia, Sol y especialmente Universidad exceden las pérdidas previstas. De hecho, Justicia y sobre todo Universidad se ven mejor reflejadas por la hipótesis «b», expresada en la columna 4 del Cuadro III, es decir, aquella que sostiene como hipótesis

${ }^{7}$ PAI (Programa de Acciones Inmediatas): Distrito Centro, vol. 10: Población: proyecciones $y$ datos complementarios, Madrid, COPLACO, estudio no publicado, noviembre 1979 , p. 3. 
las mismas tasas migratorias del quinquenio precedente. Siguiendo con el tema de las proyecciones, el PAI-Centro asumía que desde 1979 un programa de intervención pública como el que proponían los propios documentos del PAI debería acabar con la situación de sangría migratoria del distrito y, aun más, conseguir una corriente inmigratoria que lo reequilibrara. Sobre este supuesto el distrito Centro llegaría a 1985 con una población de 165.741 residentes, prácticamente la misma que en 1980. Tan optimistas previsiones venían matizadas por la consideración de la limitada capacidad de gestión de las Administraciones y las inversiones, tanto para la Administración Local, de forma aislada, como en cooperación con diversos organismos de la Administración Central o en convenio con otras fuerzas sociales. Resultaba evidente que las medidas cautelares y de tipo legal (fundamentalmente de planeamiento) habían sido puestas en práctica o podían serio sin muchas dificultades, cosa que no podía decirse de la capacidad de gestión y las inversiones, a todas luces insuficientes y en todo caso de lenta ejecución.

En función de toda esta serie de condicionantes y con objeto de establecer una cifra previsible de población para el año horizonte de 1983 se realizó una proyección en la que, tomando como base la población de 1980, se mantuviera el comportamiento frente a la fecundidad y la mortalidad (lo que es una suposición un tanto abusiva, pero necesaria si no se quiere complicar el cálculo), mientras la emigración para cada grupo de edad se redujo en un porcentaje que oscilaba entre el 20 y el $33 \%$ sobre la del quinquenio anterior (que ya se había reducido en un $20 \%$ sobre la del precedente).

A tenor de estos cálculos, y según una hipótesis moderada, la población del distrito se situarla, en 1983, en 153.087 residentes (Cuadro IV), dentro del cuadro de alternativas bosquejado, lo que sigue significando una pérdida muy elevada. La realidad, sin embargo, se ha encargado de poner en evidencia el optimismo de las previsiones más moderadas puesto que ya en el Padrón municipal de 1986, y salvada la diferencia de un año con la fecha de las proyecciones, la población había descendido hasta los 143.677 residentes, cifra mucho más próxima a la hipótesis «c», es decir, aquella que mantenía las mismas tasas migratorias del quinquenio 1975-80. Las proyecciones no avanzan hasta el último Censo de 1991, sin duda aquél en el que se consiguen las mejores cifras relativas de las últimas décadas, puesto que, aunque la población 
continúa descendiendo, el ritmo se ha reducido considerablemente, dentro de la tónica general de ralentización del proceso de despoblación de Madrid por las salidas de sus habitantes. Todo ello podría indicar, en el caso concreto del casco antiguo, un período de cierta estabilización vinculado tanto a políticas de protección y recuperación lideradas desde la Administración como a los propios cambios de estrategia del mercado inmobiliario que ha decidido recuperar el casco antiguo como lugar de residencia, entrando de lleno en los procesos de rehabilitación y reacondicionamiento y de modo más llamativo en los procesos de renovación enmascarados tras el «fachadismo» y la reestructuración total, potenciando para ello no sólo sus valores de centralidad sino también, y muy especialmente, los aspectos más emblemáticos ligados a su pasado histórico y a la imagen colectiva.

A grandes rasgos, y pese al tímido cambio de tendencia recientemente iniciado, el análisis de la crisis demográfica por la que ha atravesado y todavía atraviesa el distrito Centro sigue respondiendo al mismo análisis realizado en 1979 por el PAI-Centro, estudio que por su envergadura y características venimos citando como referencia continua en nuestra investigación. Desde el final de la década de los cincuenta, la población ha disminuido de forma acelerada bajo el doble impulso del abandono y la expulsión y sustitución por otros usos más rentables. El proceso de vaciamiento se ha visto complicado, además, por la peculiar estructura de edades de la población del distrito, cuyo grado de envejecimiento es tan notorio que la tasa de crecimiento natural no puede ser sino negativa. En origen, la reconversión de la economía española, en un esfuerzo promovido desde el Estado para acercarla a los niveles de desarrollo de la Europa occidental, tuvo nefastas consecuencias para el distrito. Los cambios en el modelo de consumo y las expectativas de localización y tipología residencial, muchas veces artificialmente suscitadas, provocaron el rechazo de la vivienda tradicional ${ }^{8}$ sin querer negar con esto que, en muchos casos, la misma no reunía unos niveles de confort mínimos. En cualquier caso, la paulatina salida de la población residente se vio acompañada por el crecimiento del sector terciario y de

8 Valenzuela Rubio, M.; Lago Ávila, M. J. y Vázquez Varela, C (1992): «Dinámicas recientes del espacio residencial madrileño», Urbanismo COAM, 16, pp. 14-26; Valenzuela Rubio, M. y Vázquez Varela, C. (1991): «Promoción cooperativa y nuevas periferias residenciales en el área metropolitana de Madrid», Actas del XII Congreso Nacional de Geografia, Valencia, 1991, pp. 625-631. 
servicios y, con ello, por la presión sobre la zona central de la ciudad, que por sus peculiares características no estaba en condiciones de responder con agilidad a los nuevos requerimientos, entrando en un proceso de crisis demográfica, deterioro y congestión que sólo en fechas recientes se ha tratado de paliar.

La pérdida de población es algo generalizado en el distrito. Todos sus barrios han experimentado importantes caídas en su número de residentes, lo cual no impide que existan diferencias significativas entre ellos. Los barrios donde el proceso de sustitución ha revestido mayor intensidad, es decir, los más terciarizados, Sol y Cortes han registrado índices de pérdida de población extremadamente altos. Por el contrario, aquellos otros en los que tanto la trama urbana, como las condiciones objetivas del patrimonio arquitectónico y su estratégica localización se acomodan mejor al uso residencial y en los que, además, el proceso de sustitución no ha sido tan fuerte han podido resistir mejor el éxodo masivo de población.

Las zonas que han perdido más población responden mayoritariamente a dos tipologías bien diferenciadas. Por una parte, el interior de los barrios populares donde tanto la densidad de población como la de viviendas muy degradadas es alta, Lavapiés y Malasaña. Y, por otra parte, áreas sometidas a un fuerte proceso de terciarización y por lo tanto de tensión especulativa: es el caso del área de la Gran Vía, Sol-Callao y el tramo del Paseo del Prado comprendido entre las Plazas de Cibeles y Neptuno, no descartándose en ocasiones la superposición de ambos fenómenos.

\section{El envejecimiento del envejecimiento}

El título de este epígrafe hace referencia al fenómeno descrito por la acumulación de efectivos poblacionales en los intervalos de edad que no sólo superan los 60-65 años, límite convencional a partir del cual se sitúa lo que comúnmente se denomina tercera edad o población anciana, sino que engrosan de forma destacada las cohortes que rebasan los 75 años de edad.

$\mathrm{Al}$ igual que ya afirmábamos al hablar de la crisis demográfica por la que viene atravesando el distrito Centro de Madrid, el proceso de envejecimiento no puede cogernos desprevenidos en tanto es uno de los 
componentes más conocidos del complejo fenómeno de la decadencia poblacional. El galopante proceso de envejecimiento que viene afectando a la sociedad española ${ }^{9}$, y especialmente a las áreas centrales de las ciudades, es consecuencia directa del progresivo incremento de la esperanza de vida (actualmente en torno a los 73 años para el hombre y a los 80 para la mujer) y, sobre todo, del fuerte descenso de la tasa de fecundidad. España es el segundo país del mundo con el índice de fecundidad más bajo (1,3 hijos por mujer) detrás de Italia (1,29 hijos por mujer). Con estas cifras queda descartado el reemplazo generacional, ya que para asegurarlo deberíamos tener un índice de fecundidad de 2,1 hijos por mujer.

El distrito Centro, al margen de los graves problemas de expulsión que han afectado prioritariamente a las cohortes de población en edad fértil, no ha sido ajeno a la evolución de las pautas reproductivas de sus residentes que, de modo paulatino, y como han demostrado numerosos estudios, han ido reduciendo el promedio de hijos por mujer desde las primeras décadas del presente siglo ${ }^{10}$. Como fácilmente se constata en el Cuadro V, la media de hijos nacidos vivos por madre desciende a medida que el año de nacimiento de ésta se aproxima a nuestros días, pasando de los 5,2 hijos por madre entre aquéllas nacidas antes de 1891 a los 1,18 hijos de media para las que nacieron después de 1975. Bien es cierto que las madres nacidas con posterioridad a 1946 mantendrían todavía, a efectos estadísticos, su capacidad reproductiva, con lo que la media sería susceptible de verse incrementada en el futuro. Sin embargo, los comportamientos antinatalistas que mantiene la población residente en el distrito no animan a esperar grandes modificaciones. De hecho, y una vez obviada la población femenina sin descendencia a la que más adelante nos referiremos, ninguno de los grupos quinquenales de madres nacidas con posterioridad a 1951 ha conseguido alcanzar la media de 2,1 hijos, cifra límite por debajo de la cual resulta inviable el reemplazo generacional, si a esto añadimos las mujeres en edad fértil

9 Rodríguez Rodríguez, V. y Rojo Pérez, F. (1989): «La evolución del envejecimiento en España desde 1900 a 1986», II Jornadas sobre la Población Española, Palma de Mallorca, pp. 381-402; Vinuesa Angulo, J. (1990): Proceso de envejecimiento de la población de Madrid, I.E.G.A., Departamento de Geografía Humana y Regional, C.S.I.C., Documentos de Trabajo, 8, $40 \mathrm{pp}$.

${ }^{10}$ Leasure, William J. (1963): «Factors involved in the Decline of Fertility in Spain, 1900-1950», in Population Studies. A Journal of Demography, XVI, 3. 


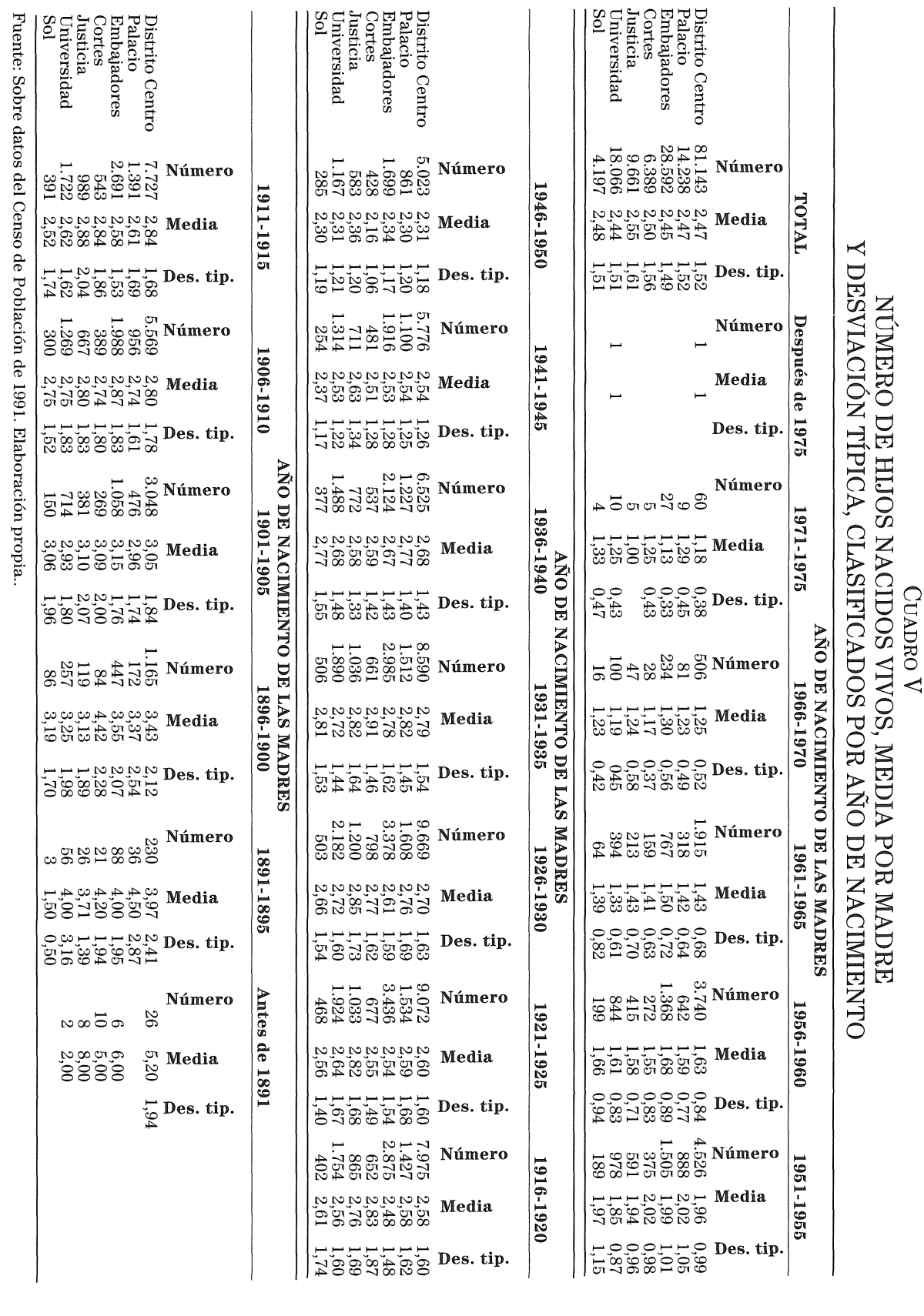

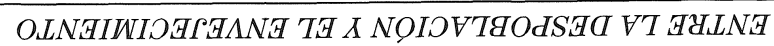


que no han tenido descendencia, comprobaremos la aguda crisis por la que atraviesan las tasas de fecundidad del distrito. Especialmente la distribución del fenómeno es muy homogénea y no se puede hablar de diferencias significativas inter-barrios. La media de hijos nacidos vivos por madre se ha reducido cronológicamente al mismo ritmo dentro de todo el ámbito de estudio.

Algunas diferencias inter-barrios pueden establecerse, sin embargo, si consideramos el conjunto de la población femenina clasificada por el número de hijos nacidos vivos (Cuadro VI). En este caso no nos interesa conocer la media de hijos por madre sino cuál es el número más fre-

CUADRo VI

POBLACIÓN FEMENINA, CLASIFICADA POR NÚMERO DE HIJOS NACIDOS VIVOS

\begin{tabular}{|c|c|c|c|c|c|c|}
\hline & \multirow[t]{2}{*}{ TOTAL } & \multicolumn{5}{|c|}{ Número de hijos nacidos vivos } \\
\hline & & 0 & 1 & 2 & 3 & 4 \\
\hline & 1991 & 1991 & 1991 & 1991 & 1991 & 1991 \\
\hline Distrito Centro & \#\#\#\#\# & \#\#\#\#\# & 9.077 & \#\#\#\#\# & 6.310 & 3.118 \\
\hline Palacio & \#\#\#\#\# & 7.314 & 1.554 & 2.040 & 1.10 & 536 \\
\hline Embajadores & \#\#\#\#\# & \#\#\#\#\# & 3.235 & 4.052 & 2.273 & 1.103 \\
\hline Cortes & 6.153 & 3.597 & 711 & 873 & 478 & 240 \\
\hline Justicia & 9.267 & 5.473 & 1.027 & 1.258 & 748 & 376 \\
\hline Universidad & \#\#\#\# & \#\#\#\# & 2.077 & 2.606 & 1.370 & 681 \\
\hline \multirow[t]{4}{*}{ Sol } & 4.155 & 2.462 & 473 & 562 & 334 & 182 \\
\hline & \multicolumn{6}{|c|}{ Número de hijos nacidos vivos } \\
\hline & 5 & 6 & 7 & 8 & 9 & $>9$ \\
\hline & 1991 & 1991 & 1991 & 1991 & 1991 & 1991 \\
\hline Distrito Centro & 1.497 & 718 & 337 & 193 & 89 & 124 \\
\hline Palacio & 282 & 116 & 52 & 33 & 15 & 24 \\
\hline Embajadores & 509 & 243 & 111 & 74 & 26 & 37 \\
\hline Cortes & 124 & 58 & 36 & 20 & 7 & 9 \\
\hline Justicia & 179 & 98 & 53 & 24 & 11 & 20 \\
\hline Universidad & 338 & 170 & 65 & 32 & 24 & 28 \\
\hline Sol & 67 & 33 & 20 & 10 & 6 & 6 \\
\hline
\end{tabular}

Fuente: Sobre datos del Censo de Población 1991. Elaboración propia.

$$
-666-
$$


cuente de descendientes entre un conjunto mucho más amplio, el del total de la población femenina del distrito, y, por otra parte, saber si esos porcentajes se repiten por igual en los seis barrios administrativos.

Los resultados del Censo de 1991 no dejan lugar a dudas: más de la mitad de la población femenina del distrito (un 56,61\%) no ha tenido ningún hijo, cifra sorprendente si consideramos que estamos hablando de una población muy envejecida y que por lo tanto acumula la mayor parte de sus efectivos en las cohortes de edad fértil o en aquellas que ya han dejado de serlo. Aunque las diferencias no son demasiado notables en términos porcentuales, sí resulta significativo que Palacio y especialmente Embajadores se distingan con proporciones de población femenina sin hijos algo más bajas que la media del distrito $(55,95 \%$ y $53,42 \%$ respectivamente), mientras Sol, Justicia y Universidad acumulan los mayores porcentajes en esta categoría. La situación se invierte al considerar el número de mujeres que sí han tenido algún hijo. Para empezar, y antes de comentar las características internas de cada barrio, diremos que el número de hijos más frecuente en todos los casos resulta ser el de dos, el 15,04\% de las mujeres del distrito ha tenido este número de descendientes, seguido por el grupo de mujeres con un sólo vástago y a continuación todos los demás grupos, descendiendo la proporción de mujeres en cada uno de ellos a medida que el número de hijos aumenta. Por barrios, las puntuaciones más elevadas, hasta llegar al grupo que recoge las mujeres con seis hijos nacidos vivos, las acaparan de forma constante Embajadores y Palacio, a partir de ese grupo, y a medida que el número de hijos aumenta, las proporciones son más homogéneas y no existen diferencias significativas entre los seis barrios del distrito, que ocupan alternativamente las primeras posiciones, reflejando lo que podría considerarse como comportamientos natalistas claramente superados o marginales.

Las conclusiones que permite extraer el análisis de los datos descritos no dejan lugar a dudas, el distrito Centro, y dentro de él cada uno de sus seis barrios, están aquejados por una grave crisis de sus tasas de fecundidad, que han venido retrocediendo de forma acelerada a lo largo de las últimas décadas. La situación, sin embargo, dista mucho de ser uniforme para todo el ámbito de estudio. La mitad norte se encuentra en una fase regresiva claramente más avanzada que la mitad sur del distrito, donde los barrios de Palacio y Embajadores, menos terciarizados y con una población de extracción socioeconómica más débil, han con- 
seguido mantener hasta la fecha unas tasas de fecundidad algo menos desequilibradas. Las perspectivas a corto plazo no arrojan mucha luz sobre el futuro de estos barrios, los elevados índices de envejecimiento, la estructura de hogares existente y de nueva creación, la tipología de viviendas en expansión (apartamentos y estudios), las características socioeconómicas de los residentes y el cambio de estilos de vida, entre otras muchas variables coyunturales y estructurales, no parecen contribuir a corto plazo a una elevación significativa de las tasas de fecundidad del distrito.

Por otra parte, y volviendo sobre las causas del envejecimiento poblacional que aquí nos ocupa, de todos es bien sabido que la pérdida de población no afecta por igual a todos los grupos de edad. Las personas que tienen mayor movilidad social o se encuentran en los momentos más críticos de su decisión residencial: los jóvenes y especialmente quienes por matrimonio u otra razón se constituyen en un hogar independiente, serán los más propensos a cambiar su lugar de residencia. Los efectos de estas pérdidas no sólo se reflejarán en las cohortes de edad de los propios migrantes sino que también tendrán su reflejo en aquellos grupos de edad a los que pertenecerían sus descendientes. Por el contrario, aquellos con menos opciones o razones para irse, en su mayoría población anciana de bajos recursos, quedarán fijados en sus residencias tradicionales. Como consecuencia de todo ello, la pirámide de población del distrito Centro (es decir, su estructura por sexo y edad) ha comenzado a verse completamente distorsionada en un proceso que el paso del tiempo sólo ha contribuido a empeorar.

La deformación de la pirámide de edades (Figura 1) refleja un proceso de «retroalimentación» que no hace sino profundizar la crisis demográfica del distrito. El número total de defunciones excede al de nacimientos y la Influencia que en cada grupo de edad tienen los procesos de expulsión y abandono contribuye a aumentar el desequilibrio demográfico ${ }^{11}$ : la población infantil cada vez es menor y cada vez mayor la de la tercera edad.

11 Pérez Sierra, C. (1990): «Áreas sociales, segregación espacial y movilidad demográfica en la Comunidad Autónoma de Madrid», Jornadas Internacionales sobre Demografía Urbana y Regional, Madrid, 17 pp.; Serrano Torrego, F. (1990): «Los movimientos migratorios madrileños en las últimas décadas y su incidencia espacial», Jornadas Internacionales sobre Demografía Urbana y Regional, Madrid, 14 pp. 

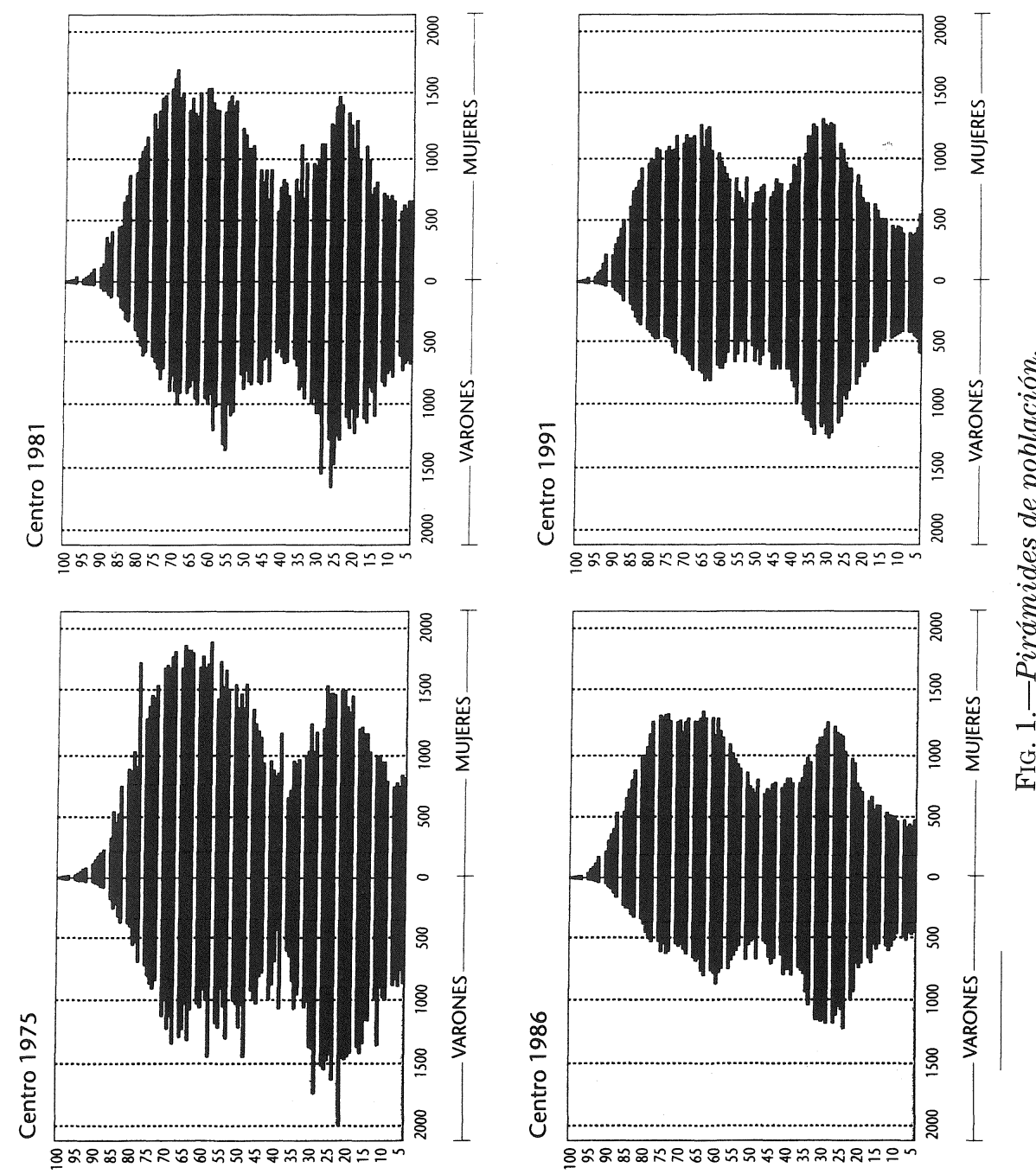
Esta situación se extiende de forma generalizada a todos los barrios del distrito; sin embargo, una visión más detallada de su distribución espacial a mayor escala presenta algunos matices significativos. En primer lugar, aquellas zonas en las que la sustitución de usos residenciales registra los índices más elevados compatibilizan frecuentemente esta situación con elevados porcentajes de población en edad avanzada (véase el caso de Sol). Y, en segundo lugar, los desequilibrios de la estructura demográfica están más atenuados en la mitad sur del casco, con la excepción del núcleo interior de Malasaña en el barrio de Universidad y algunas zonas de borde donde la renovación ha sido mayor.

Para corroborar estas afirmaciones, nada mejor que observar la estructura de población reflejada en los Cuadros VII, VIII y IX. En los mismos se compara, diferenciada por sexos y clasificada en intervalos de edad quinquenales, la población que, tanto en términos absolutos como porcentuales, arrojaron, respectivamente, el Censo de 1970, el Padrón municipal de 1986 y el Censo de Población de 1991. Aun conviniendo que el intervalo de tiempo considerado es relativamente breve en términos demográficos, las tendencias que en él se manifiestan son indudablemente significativas. Los datos de 1970 elevaban la cifra de población que entonces rebasaba los 65 años a un 16,3\% del total, quince años después, en 1986, el porcentaje ha llegado a un $24 \%$. En tan sólo cinco años, los que separan el Padrón de 1986 del Censo de 1991 y, si bien la población de ambos sexos mayor de 65 años se ha mantenido estable en un $24,3 \%$ sobre el total, lo cual es ya una cifra muy elevada, aquella que supera los 75 años de edad ha pasado de suponer un 10,92\% a un 11,63\% de todos los efectivos, cuando este mismo grupo sólo sumaba un modesto 5,75\% en 1970. Estos datos ilustran el proceso cuyo nombre da título a este epígrafe y que a grandes rasgos se repite en todos los barrios con pequeñas variaciones.

En 1970, Sol se distanciaba con las mayores tasas de envejecimiento del distrito (18,13\%), seguido por Cortes y Justicia (17,5\% y $17 \%$ respectivamente) mientras Universidad, Palacio y Embajadores ocupaban los últimos puestos en esta jerarquía (16,68\%, 15,71\% y 15,38\% por este mismo orden), relación que se mantenía inalterada en el colectivo de residentes mayores de 75 años. En 1986, y si bien Sol se mantenía a la cabeza de la lista de los barrios más envejecidos (el $26,27 \%$ de su población superaba los 65 años), no ocurría lo mismo con Cortes y Justicia, que habían cedido sus puestos a Universidad y Embajadores respecti- 


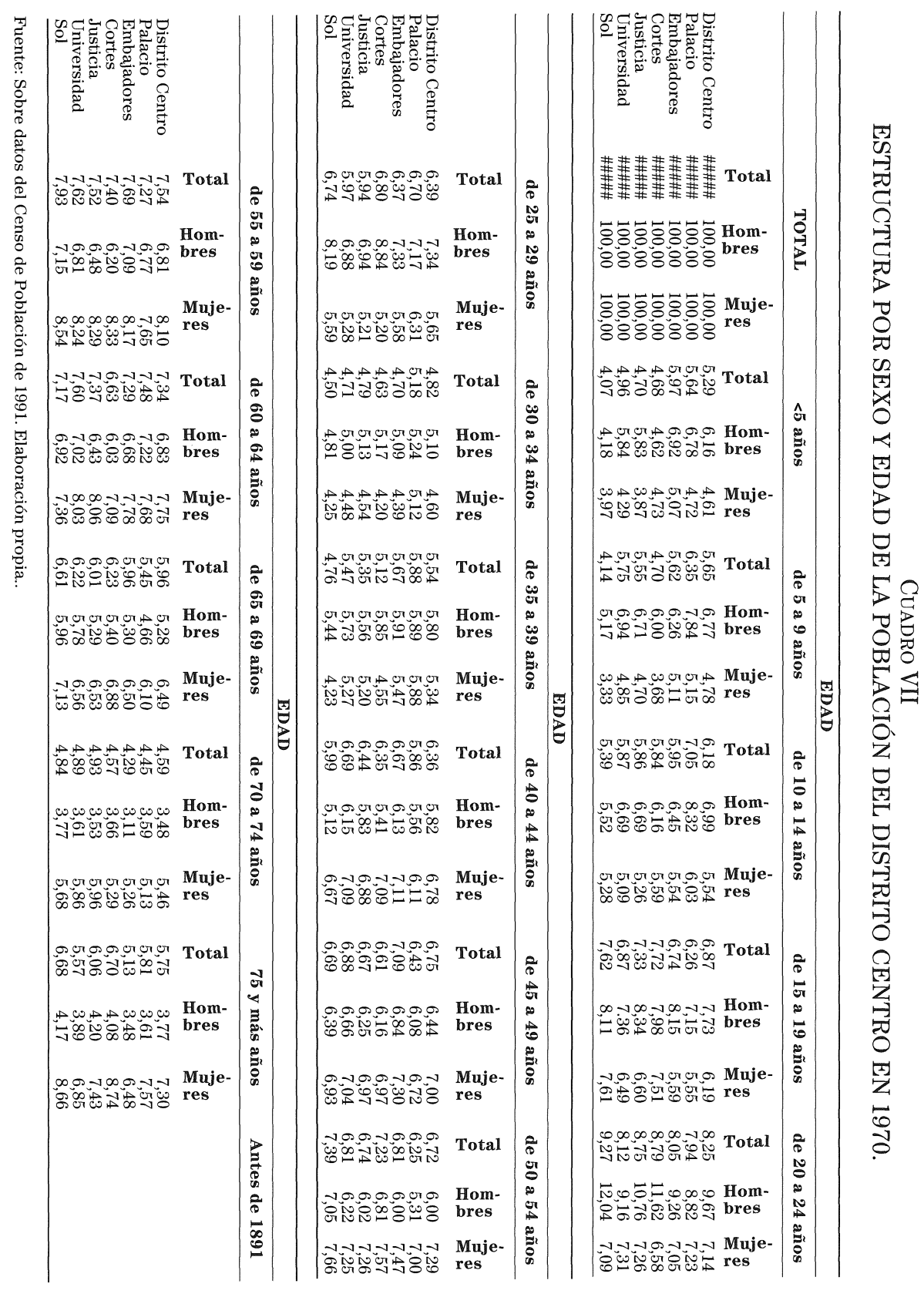

OLNAILIOHГAЯ 


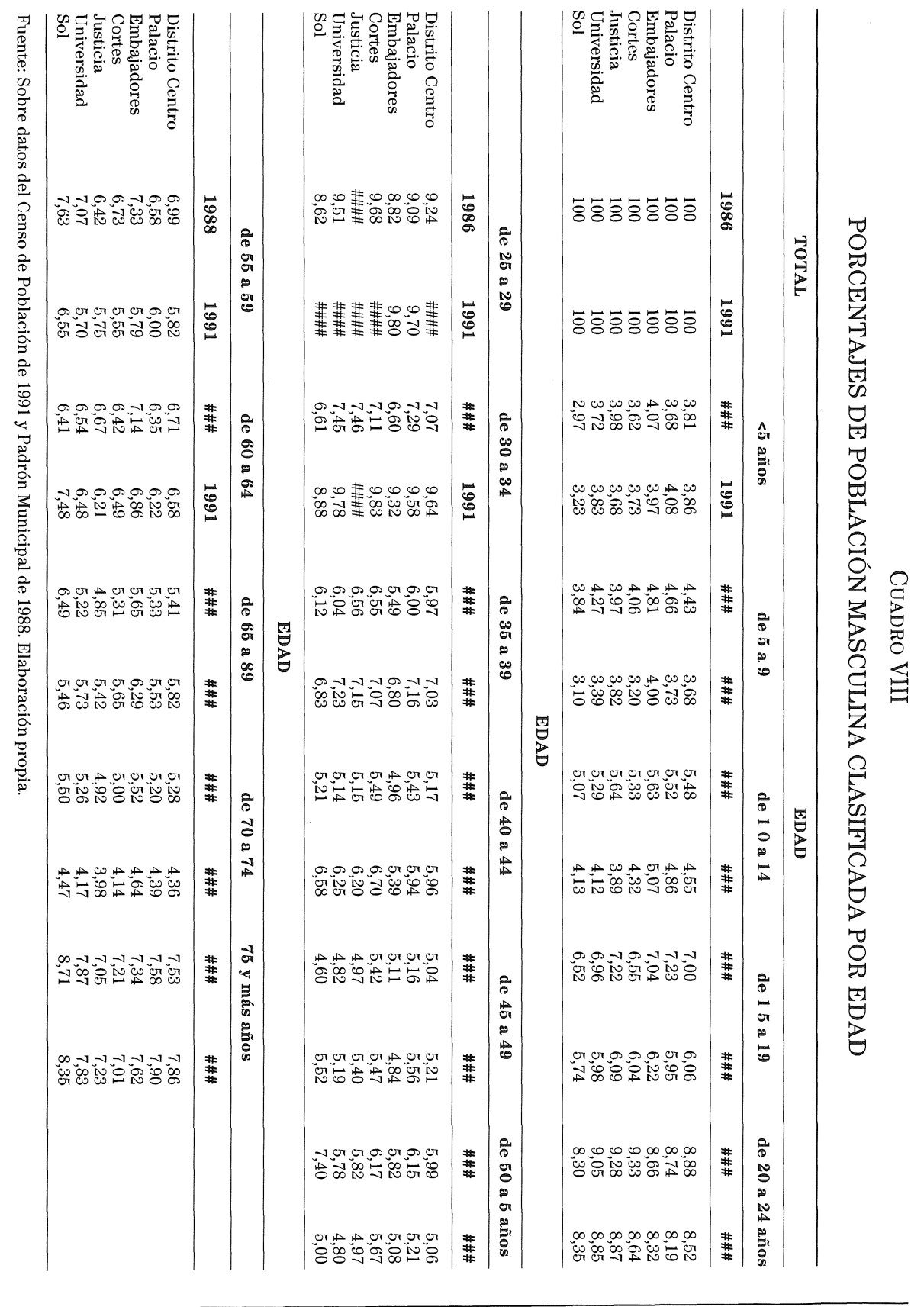

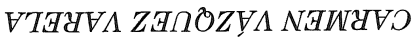




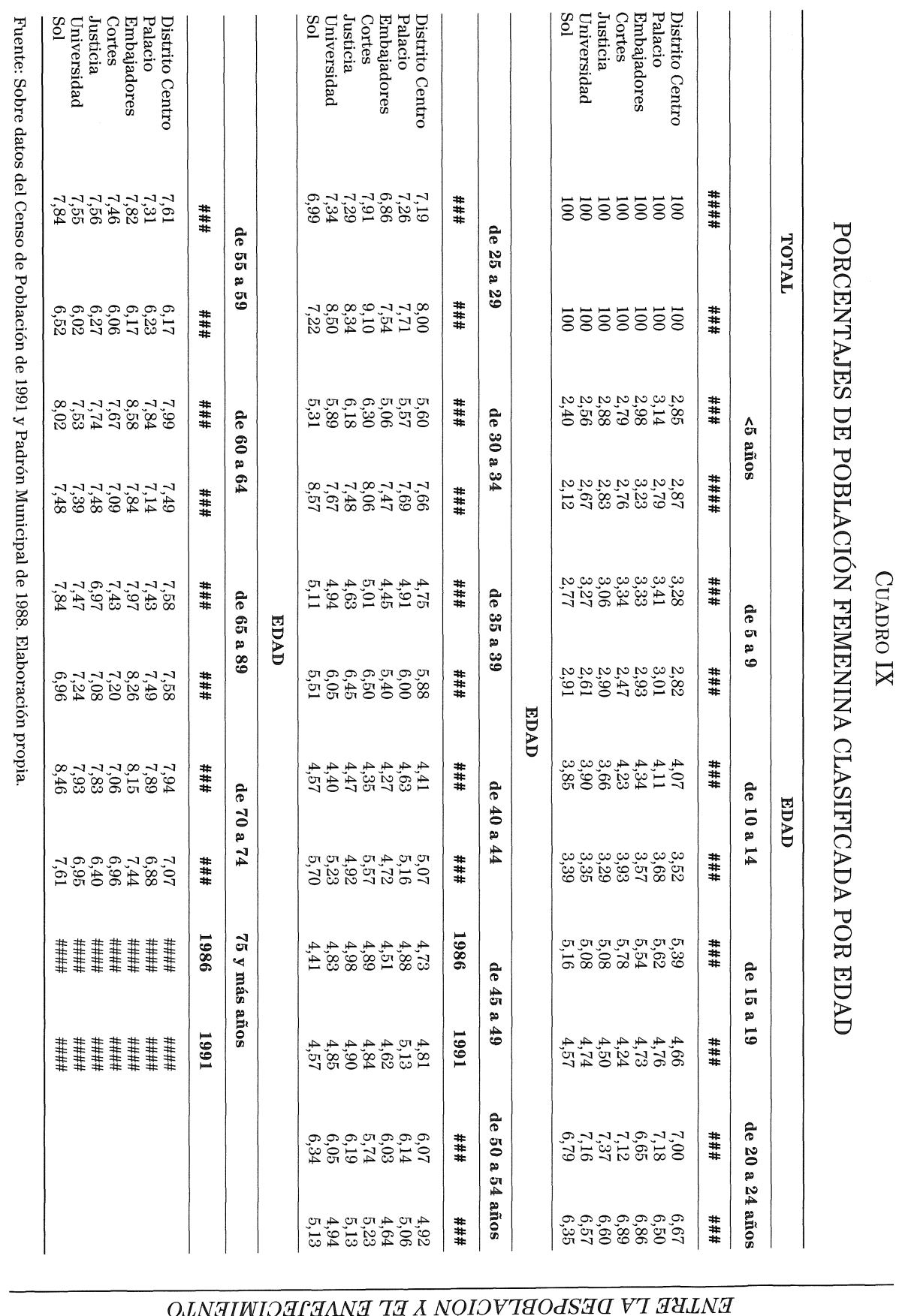


vamente $(24,70 \%$ y $24,69 \%)$, barrios que hasta entonces habían disfrutado de una composición por edades algo más equilibrada, Palacio, Justicia y Cortes les seguían en este proceso de envejecimiento imparable, todos ellos con tasas superiores al $22 \%$ del total de efectivos poblaciones. En el último recuento de población, realizado en 1991, tanto Cortes, como Justicia y Universidad se han beneficiado de un leve decrecimiento de sus residentes mayores de 65, mientras Palacio y Embajadores, por el contrario, han visto incrementados sus índices (Fig. 2). Mención aparte merece el caso de Sol, tradicionalmente un barrio muy terciarizado y extremadamente envejecido que hasta 1986 ostentaba el primer lugar en la clasificación de barrios por grado de envejecimiento, en tan solo cinco años ha descendido su porcentaje en casi dos puntos pasando de un $26,27 \%$ a un $24,85 \%$ y ocupando ahora el segundo lugar tras Embajadores (25,07\%) en razón de sus proporciones de población anciana.

El comportamiento de la población mayor de 75 años es una copia casi exacta del anterior: en 1970 la jerarquía inter-barrios seguía fielmente las pautas marcadas por el grupo genérico de la tercera edad (mayores de 65 años) con la poco relevante excepción del intercambio de puestos entre Cortes (que ahora encabezaba la lista en términos porcentuales) y Sol. En 1986 la clasificación era de nuevo un calco de la de este mismo grupo al realizarse el empadronamiento, salvo otro pequeño trasvase de puestos entre Justicia y Embajadores, respectivamente el segundo y tercer puesto. Por último, en 1991, y a diferencia de lo ocurrido con la clasificación general del colectivo de ancianos (Fig. 3), Sol sigue manteniendo la primacía, mientras todos los barrios, con la sola excepción de Cortes, ven incrementada su representación respecto a los datos del Padrón de 1986.

Los intervalos de población más joven, es decir, y a efectos de nuestro análisis, aquéllos con una edad inferior a los 15 años, experimentan pérdidas de desigual cuantía, con la sola excepción de los menores de 5 años que, en el último quinquenio, logran estabilizarse e incluso aumentar levemente en algunos barrios. La pérdida de efectivos de edad infantil y juvenil parece ser crónica y ni siquiera el sutil incremento del primer escalón de la pirámide es motivo suficiente para predecir una estabilización de la estructura de población por sexo y edad a corto plazo.

Una situación bien distinta es la planteada por el incremento de los efectivos, en términos relativos, de las cohortes de población compren-

$$
-674-
$$




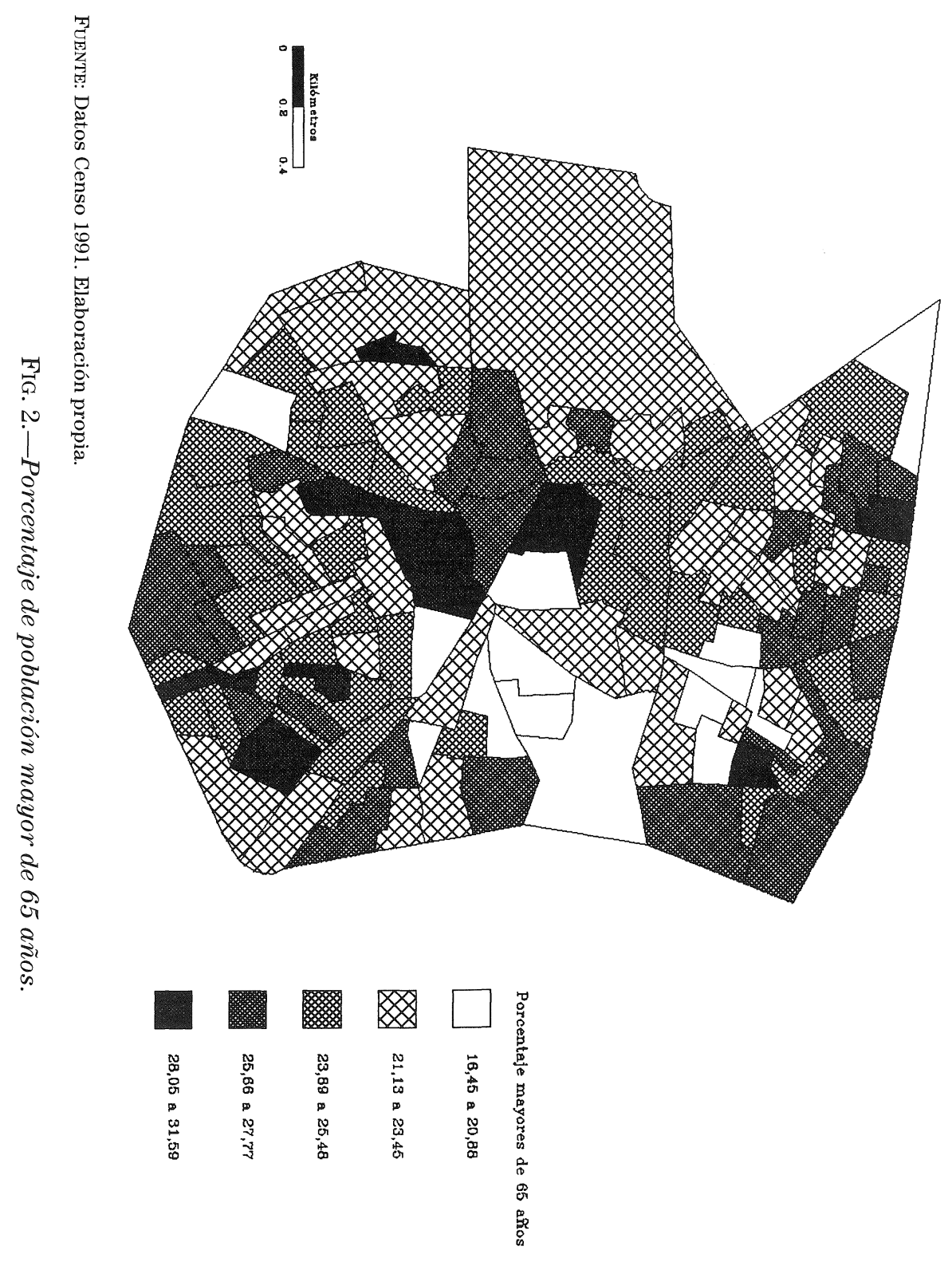

OLNGIWIDGPA ANA TH X NOIOVTGOdSAG VT GYLNG 


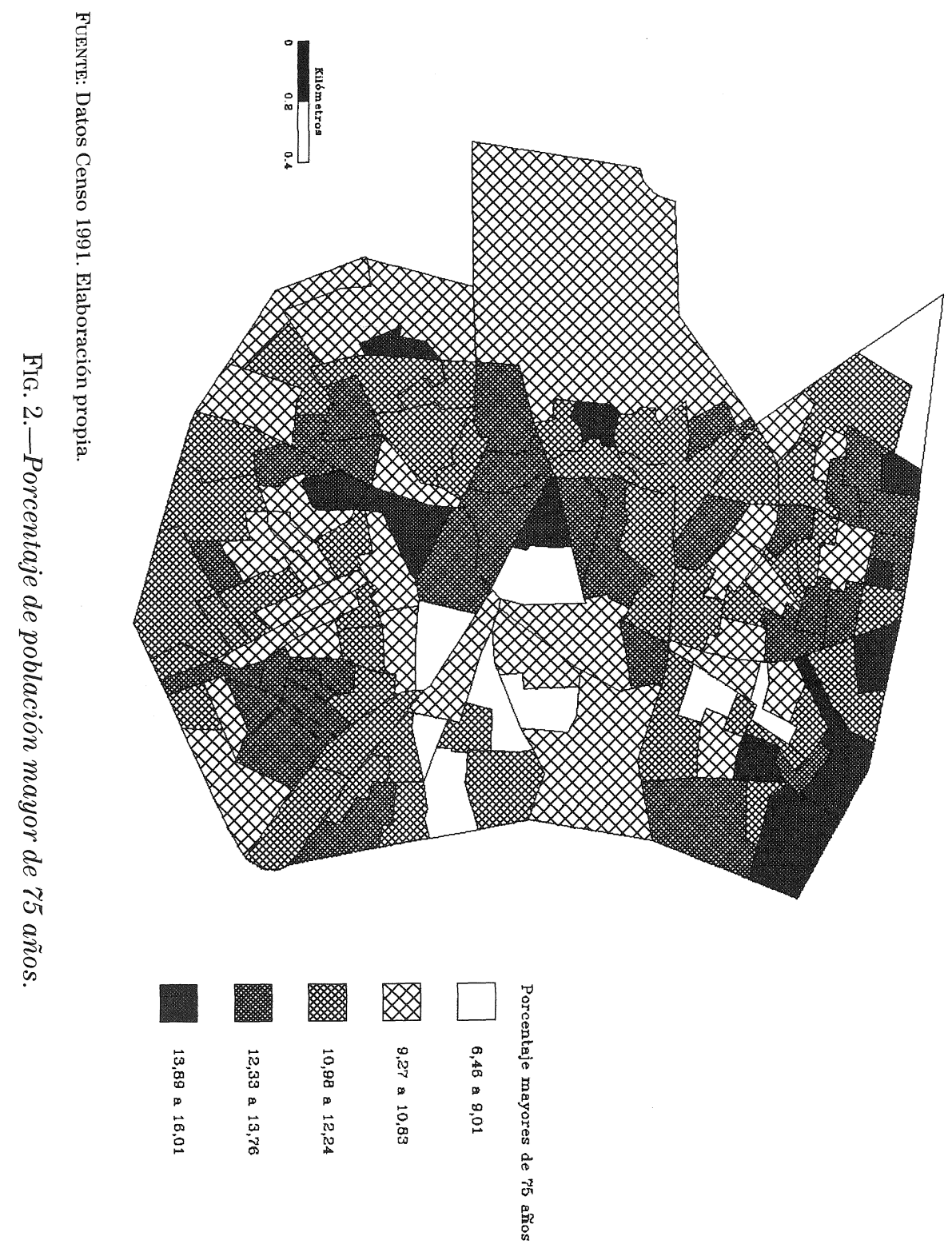

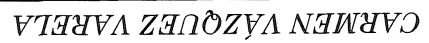


didas entre los 25 y los 34 años de edad entre 1970 y 1986, es decir, aquellas que se situarían en el primer ciclo productivo, incremento que se mantiene entre 1986 y 1991, afectando ahora a los residentes entre 25 y 44 años de edad, tanto en términos absolutos como relativos. En este último caso todo parece indicar que el incremento es fruto de aportes externos ya que la dinámica interna del propio distrito, regresiva desde hace varias décadas, ha sido incapaz de generar unos aumentos tan significativos y uniformes como resultado de su ritmo de sustitución generacional. Si bien parte de los mismos pudieran atribuirse en principio a las generaciones nacidas durante el «baby-boom», este hecho no explicaría lo sucedido con los residentes comprendidos entre los 35 y los 44 años de edad, que vieron la luz antes de la citada eclosión demográfica.

Si admitimos, pues, que gran parte de esos incrementos en las cohortes de población adulta se corresponden con procesos inmigratorios que tienen como destino el centro histórico de la ciudad, quedaría por determinar cuáles son las causas que los provocan y, en consecuencia, qué características reúnen los nuevos residentes del casco antiguo. El deterioro progresivo del espacio construido del distrito ha provocado la salida de muchos de sus residentes tradicionales, pero también ha constituido un foco de atracción para grupos de población vulnerables y en muchos casos claramente marginales que han encontrado en el mismo la única posibilidad de acceder a una vivienda con la ventaja añadida de su posición central. Entre estos grupos marginales y empobrecidos se cuenta, desde hace algunos años, un buen número de inmigrantes, especialmente africanos, que, en situación de legalidad o ilegalidad, han hecho del distrito Centro su lugar de trabajo y residencia. En cualquier caso, la tipología de grupos marginales que van colonizando paulatinamente el espacio interior, y de la que más tarde nos ocuparemos, es muy amplia y poco a poco todos estos grupos han encontrado en los intersticios de la ciudad un lugar donde reproducir sus propios conflictos $^{12}$.

Por otra parte, y frente a estos grupos de población marginales, se ha ido produciendo a lo largo de los últimos años la llegada, mucho más selectiva especialmente y reducida en efectivos, de segmentos de pobla-

12 Giménez Romero, C. (Coord.) (1993): Inmigrantes extranjeros en Madrid, Madrid, Secretaría General Técnica, Consejería de Integración Social, Comunidad de Madrid, 2 vols. y López García B. et al. (1993): Inmigración magrebi en España. El retorno de los moriscos, Madrid, Ed. MAPFRE, Colección El Magreb, 428 pp. 
ción solvente compuestos en su mayor parte por hogares de tamaño reducido, una o dos personas, con niveles de estudios medio-alto y elevada cualificación profesional. Las razones de este regreso al interior de los cascos urbanos hay que buscarlas en un complejo entramado de nuevas demandas (culturales, sociales, de ocio, etc.) y estilos de vida que se reflejan en la composición cuantitativa y cualitativa de los hogares de reciente creación en el distrito y en las tipologías residenciales en expansión. Pero tampoco hay que olvidar el poderoso influjo del mercado inmobiliario y de los intereses de los agentes urbanos en esta nueva puesta en valor de un espacio largo tiempo olvidado y que de nuevo recupera su atractivo merced a las sustanciosas plusvalías que ofrece su recuperación.

Por último, y antes de pasar a analizar las consecuencias del acelerado proceso de envejecimiento poblacional que aquí nos ocupan, hablaremos del grado de incidencia del mismo en función del sexo de los residentes. Dado que líneas atrás hemos examinado detenidamente la distribución de los efectivos en función de los grupos de edad en que se inscriben y los cambios experimentados en el breve espacio de las dos últimas décadas, poco más vamos a añadir ahora al respecto ya que al perfil dibujado se ajustan igualmente tanto los varones como las mujeres con una única pero importante distinción. Manteniendo la misma distribución del peso por grupos de edad, los varones equilibran el reparto traspasando parte de la carga a las cohortes de edad más joven, con lo que la proporción de hombres mayores de 65 años, que en 1970 se elevaba a un $12,53 \%$, supone, en 1991, «solamente» un $17,84 \%$, y la de aquellos que superan los 75 años de edad pasa del $3,77 \%$ a un modesto 7,66\%. Además, los cinco años transcurridos entre 1986 y 1991 no han supuesto un mayor envejecimiento de los efectivos varones sino todo lo contrario, la población masculina mayor de 65 años ha descendido proporcionalmente, pasando de suponer un $18,22 \%$ al ya mencionado 17,84\%, no así el grupo de mayores de 75 años, que sí ha registrado un leve incremento. Por barrios, al igual que el caso del conjunto de la población de ambos sexos, el primer puesto, por grado de envejecimiento, lo ocupa en 1991 Embajadores, seguido de Sol que ha perdido la primacía que ostentaba en 1986.

En el caso de las féminas la situación es bien distinta. Conservando la misma distribución general por grandes grupos de edad, el peso de la población anciana mayor de 65 años se incrementa desde un 19,25\% en 
1970 hasta alcanzar el 29,45\% en 1991 sobre el total de mujeres del distrito, mientras aquellas que superan los 75 representan ahora el 14,80\%, frente al 7,30\% que ostentaban veinte años antes. En esta ocasión, el tiempo transcurrido ha supuesto aumentos espectaculares tanto para el grupo de mayores de 65 años como para el que ya ha cumplido los 75 . La distribución inter-barrios continúa sin variaciones.

La lectura que se deduce de los datos expuestos es muy clara: en contra de la tendencia general de envejecimiento del distrito, el conjunto de efectivos masculinos se mantiene estancado e incluso experimenta en términos porcentuales un leve rejuvenecimiento. Las causas de este fenómeno han de buscarse en la combinación de varios factores entre los que destacan, por una parte, las diferencias en las tasas de crecimiento de los diferentes grupos de edad debidas, esencialmente, a la propia estructura por edad de la población, ya que las generaciones más antiguas son también las más numerosas, y, por otra, la entrada de nuevos residentes nutriendo las cohortes de edad intermedia que parecen haber estado constituidas en buena parte por varones.

Más adelante nos detendremos, con el apoyo de material cartográfico, en la distribución espacial de la población anciana del distrito, de la que hasta el momento sólo hemos dado unas breves pincelados, profundizando un poco más en las causas de la misma. Pero por el momento querríamos continuar con una descripción de la problemática general que suscita este colectivo ${ }^{13}$.

Uno de los hechos que hasta el momento no hemos mencionado, pero que reviste especial importancia en este caso, es el de que gran parte de estos ancianos constituye hogares de reducido tamaño al vivir solos o en pareja, aspecto que perjudica en buena medida su calidad de vida, ya que muchos de ellos requerirían de algún tipo de asistencia personalizada a través de la asistencia domiciliaria o bien de su traslado a algún tipo de alojamiento tutelado donde, manteniendo su autonomía personal, pudieran disfrutar de los cuidados médicos y asistenciales básicos. Sin embargo, cualquiera de las dos posibilidades está fuera del alcance de la mayoría de la población anciana del distrito, la asistencia o ayuda domiciliaria, actualmente víctima de los recortes presupuestarios, mantiene

13 Un riguroso análisis de las condiciones y problemas que afectan a las personas que envejecen en Madrid puede verse en: López Jiménez, J. J. (1993): El envejecimiento y las personas ancianas en Madrid, Área de Servicios Sociales, Ayuntamiento de Madrid, 358 pp. 
unos estrictos baremos de acceso a fin de orientar su acción a los sectores más necesitados. Las listas de espera continúan engrosándose sin que el Ayuntamiento pueda responder con la celeridad y los recursos necesarios a todas las peticiones recibidas. Respecto a los pisos tutelados, sólo se cuenta de momento con una experiencia piloto, que, si bien parece exitosa, está muy lejos de haber cubierto una mínima parte de la demanda latente. Ante esta situación muchos de los ancianos residentes se ven obligados a ingresar en las listas de espera de alguna residencia pública cuando sus ingresos no les permiten optar por un centro privado, mientras malviven en sus domicilios en condiciones higiénico-sanitarias deplorables.

La dinámica de envejecimiento del distrito, con las consecuencias de vulnerabilidad social y marginación que la misma lleva aparejada, por incidir sobre una población de escasos recursos, ha obligado al propio tejido social a tomar cartas en el asunto para tratar de paliar una situación que los Servicios Sociales públicos sólo han logrado resolver en una mínima parte. Aunque no es éste el lugar adecuado para hablar de las iniciativas tanto públicas como privadas desarrolladas en materia de Servicios Sociales para la atención del colectivo de población anciana del distrito, mencionaremos a título de ejemplo alguno de estos proyectos como muestra del grado de concienciación alcanzado en los últimos años por los propios residentes.

Buena prueba de ello la constituye una de las más recientes actividades emprendidas desde la Federación Regional de Asociaciones de Vecinos de Madrid, que, tras desarrollar durante los años noventa y noventa y uno un servicio de información telefónica general para todos los ciudadanos de la Comunidad de Madrid, inició en el año noventa y dos un servicio específico denominado «Teléfono de Información a la Tercera Edad». Se trata de un instrumento de orientación, ayuda y seguimiento a las personas de la tercera edad, para intentar trasmitir seguridad y tranquilidad al usuario y a su familia. Asimismo es un servicio de carácter preventivo que establece una relación periódica con las personas mayores para prevenir situaciones de desatención y ser un enlace entre las necesidades y los recursos sociales adecuados.

La implantación territorial de este servicio es fiel reflejo del grado de incidencia que el proceso de envejecimiento ha alcanzado en el área. Aunque en un principio su sede se encuentra en la Federación Regional de Asociaciones de Vecinos de Madrid, cuenta en la actualidad con la co- 
laboración directa de dos Asociaciones de Vecinos ubicadas una en el distrito Centro y otra en el distrito de Chamberí («La Corrala» y «El Organillo»).

Para terminar con algunas de las consecuencias más importantes del proceso de envejecimiento del distrito, y antes de pasar a ocuparnos del tema de la población dependiente, que tantas polémicas esta suscitando al poner de relieve la fragilidad de nuestro sistema de cobertura social ${ }^{14}$, hablaremos de los desajustes generados entre tipologías de vivienda tradicional y hogares de reducido tamaño compuestos por personas de avanzada_edad. Si bien es cierto que la vivienda tipo del distrito responde en líneas generales a un modelo de reducida superficie (entre 30 y $60 \mathrm{~m}^{2}$ ), tampoco puede negarse que en el mismo existe un conjunto significativo de viviendas de gran tamaño, especialmente en su sector noreste. En un buen número de casos estas viviendas están ocupadas por personas ancianas que viven solas o en pareja y que no necesitarían de espacios tan grandes, pensados en origen para acoger a familias mucho más amplias, y cuyo mantenimiento supone en muchos casos una pesada sobrecarga para sus actuales ocupantes. A ésta se añade la frecuente ausencia del adecuado nivel de instalaciones (agua caliente, calefacción, etc.), especialmente necesarias cuando los ocupantes son personas ancianas con escasa movilidad y que por lo tanto se ven obligadas a permanecer en su domicilio de forma casi permanente. La antigüedad de las viviendas y la carencia de los recursos económicos necesarios para solventar estas deficiencias hacen imposible para la mayoría de los inquilinos o propietarios de avanzada edad afrontar cualquier tipo de reforma o mejora.

Por último, y en directa relación con lo anteriormente expuesto, nos encontraríamos con el problema del grado de abandono de la trama

14 Artajo de No, N. (1989): «Tercera Edad y pensiones públicas» (en) La Tercera Edad en Europa: necesidades y demandas, Madrid, Ministerio de Asuntos Sociales, pp. 231241; Cruz Roche, I. (1987): «La reforma de las pensiones en 1985», Economistas, 17, pp. 197-199; Desdentado Bonete, A. (1985): «La reforma de los regímenes de pensiones y su conexión con los niveles no contributivos de protección», Relaciones Laborales, pp. 1429; Durán Heras, A. (1982): «Pensiones en España: presente y proyecciones de futuro», Papeles de la Economía española, pp. 264-286. O.I.T. (1989): La protección de la vejez por la Seguridad Social, Estudio general de la Comisión de expertos en aplicación de Convenios y Recomendaciones, Ginebra, Conferencia Internacional de Trabajo, 174 pp. Paniagua Mazorra, A. (1989): «Pensiones públicas y pobreza en la Tercera Edad», Documentos de trabajo del Departamento de Geografía Humana y Regional, C.S.I.C., 24 pp. 
residencial. Aunque en este caso las variables que concurren son muy complejas (régimen de tenencia, Ley de Arrendamientos, fenómenos especulativos, etc.) no puede negarse la influencia que el grado de envejecimiento ha tenido en el problema. La escasez de recursos económicos y la elevada edad de los residentes, ya sean inquilinos o propietarios, son un factor limitante decisivo. Aun en el supuesto de que estos últimos estuvieran dispuestos a afrontar la realización de algún tipo de mejora en su inmueble o vivienda, sus características personales les vedarían el acceso para la obtención de cualquier crédito en una institución bancaria.

\section{La población dependiente}

Estadísticamente se entiende como población dependiente o índice de dependencia demográfica aquél que resulta de la relación entre el peso de los que por edad están en situación de inactividad, jóvenes y viejos, y aquéllos en edad activa (entre los 15 y los 64 años). Como bien se han encargado de señalar alguno autores (Fernández Cordón, 1993), este índice de dependencia es puramente demográfico y no responde adecuadamente al problema planteado ya que la carga debería incluir a todos los no ocupados en la producción, inactivos (aunque estén en edad de trabajar) y desempleados, que gravitan sobre los ocupados.

En términos generales, el peso de los adultos de 15 a 64 años desciende entre 1970 y 1991, tanto en cifras absolutas como relativas, pasando de un $66,58 \%$ a un $65,2 \%$. Sin embargo, el período de los últimos cinco años ha sido testigo de una cierta estabilidad con tendencia al alza, tal y como puede verse en las figuras ya mencionadas. Los porcentajes a escala de distrito pasan de representar un 63,93\% en 1986 al ya mencionado $65,2 \%$ en 1991, si bien en valores absolutos la cuantía de la población desciende pasando de 91.857 personas en 1986 a 88.769 en 1991, todo ello sin notables diferencias inter-barrios. Por su parte, los efectivos de población infantil y juvenil, que en 1970 suponían un 17,12\% se reducen a un 11,74\% en 1986 para caer en 1991 hasta situarse en un modesto $10,49 \%$.

Los resultados del último Censo no dejan lugar a dudas sobre el estancamiento experimentado por la población del distrito. A pesar del leve aumento experimentado, en los últimos cinco años, por el segmento de población de edad adulta, y de que por lo tanto el índice de de- 
pendencia demográfica ha disminuido ligeramente, la carga total por adulto, aun siendo ahora inferior, también es distinta al ser mayor el peso relativo de la población anciana y menor el de los jóvenes. En estas circunstancias, y eludiendo cualquier tipo de catastrofismo sobre las consecuencias de este proceso demográfico, parece llegado el momento de enfrentar los problemas, sobre todo los de índole económica y de integración social, que afectan de forma directa a los miembros más envejecidos de nuestra población residente y de modo indirecto al resto de la sociedad.

En vista de que la evolución demográfica y la protección social aparecen cada vez más ligadas, debido al traspaso de una solidaridad familiar y vecinal informal, a una cobertura social institucionalizada, los aspectos económicos derivados del peso de la población dependiente emergen como una de las cuestiones más debatidas en los últimos tiempos. La austeridad presupuestaria propugnada por el Gobierno para afrontar la crisis económica y los desequilibrios estructurales de nuestra economía han afectado de forma preocupante a los presupuestos en materia de Servicios Sociales ${ }^{15}$. Pero los problemas van más allá de una mera situación coyuntural y las dudas se ciernen sobre la propia capacidad del Estado para seguir manteniendo en un futuro el sistema de pensiones existente hoy en día ${ }^{16}$.

Simultáneamente, el grupo de población de 65 o más años, convencionalmente acuñado como el de los ancianos o más eufemísticamente denominado tercera edad, se disgrega en un conjunto cada vez menos homogéneo. Las mejoras en la calidad de vida, en la alimentación, en la higiene o en la atención sanitaria, unidas al alargamiento de la vida y a la generalización de la jubilación como máximo a los 65 años han ge-

${ }_{15}$ Gonzalo Fernández, B.; Ferreras Alonso, F. y Tejerina Alonso, J. I. (1985): Evolución y tendencias de la Seguridad Social durante la crisis económica, Madrid, Ministerio de Trabajo y Seguridad Social, 248 pp.; Seguridad Social (1985a): Documento de base sobre la reforma de la Seguridad Social para la Comisión tripartita del Acuerdo Económico y Social, Madrid, Ministerio de Trabajo y Seguridad Social, 152 pp.; Seguridad Social (1985b): Análisis económico-financiero del sistema español de Seguridad Social, Madrid, Ministerio de Trabajo y Seguridad Social, $282 \mathrm{pp}$.

${ }_{16} \mathrm{El}$ informe Abril, un intento de un grupo de expertos por abordar la racionalización del sistema monetario, intranquilizó a más de uno en cuanto al mantenimiento del grado de protección del Estado. Dicho informe no tenía un carácter vinculante, se trataba de una consideración de expertos, eminentemente técnica que, como tal, no tenía en cuenta algunas de las circunstancias de los pensionistas. Estas circunstancias, sin embargo, sí deben ser atendidas por los políticos, y no sólo por razones electorales. 
nerado una segmentación de este colectivo, en el que coexisten jubilados en perfecto estado de salud y con plena capacidad con personas de mayor edad, más dependientes del sistema sanitario, y con verdaderos ancianos que no pueden valerse por sí mismos. Algunos autores toman como umbrales indicativos los 75 años, a partir de los cuales se intensifica el deterioro de la salud, y los 85 como inicio de la etapa de pérdida de autonomía. El grupo de los 65-74 años plantea sobre todo un problema de integración en la sociedad, al tratarse de personas con plena capacidad pero que han salido del mundo laboral, y pueden sentirse aislados de las otras generaciones. La solución a los problemas de este colectivo exigirá de la sociedad sin duda más imaginación que recursos. Los grupos más viejos son los que ejercen mayor presión sobre el gasto público en sanidad y servicios sociales.

Una vez aceptado que cada uno de estos subgrupos exige respuestas distintas, es importante que en toda la problemática de la Tercera Edad, y particularmente en la puesta en práctica de políticas públicas, se tengan en cuenta las diferencias que el tiempo introduce en la composición interna del grupo de los mayores, en el sentido de una intensificación del envejecimiento puesto que son los más viejos los que tanto en términos absolutos como relativos más se incrementan. En las últimas décadas el grupo de mayores de 75 años ha pasado de acumular una cifra de 13.302 personas en 1970 , que representaban el $5,75 \%$ sobre la población total del distrito, a los actuales 15.830 residentes de la misma edad que suponen un porcentaje del 11,63\% (datos del Censo de 1991). Mientras tanto, el grupo de los 65-74 años (24.401 habitantes en 1970 frente a 17.240 en 1991) ha pasado de un 10,55\% en el Censo de 1970 al actual 12,67\% (datos de 1991), es decir, al tiempo que la población mayor de 75 años no sólo aumentaba en cifras absolutas sino que duplicaba su representación en términos relativos dentro de la población del distrito, el segmento de población comprendido entre los 65 y los 74 años, aquellos con menores problemas de movilidad y autonomía, descendían en número y sólo la pérdida de población generalizada que aqueja al distrito y que afecta en mayor medida a las cohortes de población más joven ha podido evitar que, proporcionalmente, su peso no sólo no descienda sino que se incremente en algo más de dos puntos.

Es evidente que los ancianos requieren una aproximación demográfica diferenciada, pues tienen sus propios índices de mortalidad, sus propios problemas de patología e incapacidad y, por tanto, de ne- 
cesidad de apoyo. De estas afirmaciones se deduce fácilmente que es indispensable conocer la situación y circunstancias de la población de edad avanzada para suministrar servicios eficaces con su costo, y planificar estrategias de intervención y asistencia. El supuesto traslado de gastos sociales de los jóvenes (de 0 a 15 años) hacia las personas de 65 y más años no es en modo alguno neutro: a tenor de los estudios realizados, una persona mayor supone tres veces más gasto social que una joven, y la fracción de este soporte que corresponde a la sociedad es casi el doble comparado con el de los jóvenes. Buena prueba de ello es que la ocupación hospitalaria está protagonizada en un $25 \%$ por las personas de 65 y más años.

Capítulo aparte merece el tema de la infraestructura de equipamientos necesarios para atender al colectivo de población dependiente. El conjunto de equipamientos a que aquí nos estamos refiriendo abarca un repertorio de servicios entre los que se cuentan los de tipo educativo, sanitario, asistencial, cultural y deportivo que facilitan la satisfacción de ciertas necesidades individuales, familiares y comunitarias de la población, principalmente niños, ancianos y grupos marginales. La peculiar composición demográfica de la población residente explica que, en el caso del equipamiento educativo, exista un exceso de oferta especialmente acusado en los casos de BUP, COU y FP, a pesar de que los centros de este tipo no son para uso exclusivo del distrito, pues existe un porcentaje de alumnos significativo procedente de otros lugares de Madrid. No obstante, este desequilibrio entre la población escolar actual y las plazas educativas no es tan grande como a primera vista parece, pues muchos de los locales no cumplen la normativa establecida por el MEC y deberán ser adaptados a la LOGSE, y otros, por su mal estado de conservación y falta de cumplimiento de normativa de seguridad e higiene, deberán ser sustituidos. Además, y en contra de lo que podría suponerse, la oferta de guarderías no cubre toda la demanda existente en el distrito.

Pero si con referencia a la población infantil y juvenil las deficiencias en infraestructura no exigirían excesivas inversiones, no puede decirse lo mismo de aquellas que afectan al multitudinario grupo de la tercera edad. En este caso, y si bien las infraestructuras en materia de Centros de Día y Clubs sociales estarían relativamente cubiertas, se constata una alarmante escasez en cuanto a pisos tutelados y residencias asistidas donde los usuarios tuvieran la oportunidad de alojarse sin necesidad de 
dejar el barrio y, por lo tanto, el entorno de redes sociales en el que siempre han vivido.

A modo de resumen, y para concluir con este apartado, podríamos decir que, a pesar de que en los últimos años, en términos cuantitativos, el peso de la población dependiente parece haberse estabilizado, cualitativamente la situación no ha hecho sino agravarse al cambiar la composición interna de ese mismo colectivo. Mientras el peso de las cohortes de población infantil y juvenil es cada vez menor, los ancianos constituyen día a día una carga más pesada ya que no sólo han aumentado su representación como colectivo sino que internamente su distribución se ha visto modificada por el acelerado crecimiento del grupo de residentes mayor de 75 años (el ya mencionado envejecimiento del envejecimiento) frente a aquéllos cuya edad se sitúa entre los 65 y los 74 años, con el consiguiente incremento de costes asistenciales y sanitarios que este proceso lleva consigo.

\section{La feminización de la estructura demográfica}

La vinculación entre envejecimiento y feminización de la estructura de población, especialmente en las cohortes de edad adulta y anciana, es un fenómeno ampliamente divulgado a través de multitud de estudios sobre el comportamiento de poblaciones de países industrializados que han pasado por el complejo proceso de transición demográfica en el que nosotros aún nos hallamos inmersos ${ }^{17}$.

De modo genérico, podría decirse que la relación de masculinidad por edades de una población viene determinada en primer lugar por dos constantes demográficas muy generalizadas, una sobremortalidad masculina, presente desde el inicio de la vida, y que aumenta con la edad, y el hecho de que nacen siempre más varones que mujeres (entre un $5 \%$ y un $7 \%$ más). Como consecuencia de estos dos factores suele haber más hombres que mujeres entre las cohortes de población más joven y más mujeres que hombres entre las más envejecidas. El otro factor que habitualmente viene a alterar esta relación son las migraciones, general-

17 Kunzmann, Klaus, R.; Wegener, Michael (1990): The pattern of urbanisation in Western Europe, 1960-1990, Institut für Raumplanung, Universität Dortmund; Parkinson, Michael et al. (1992). Urbanisation and the function of cities in the European Community, European Institute of Urban Affairs, Liverpool John Moores University.

$$
-686-
$$


mente selectivas en relación al sexo, sobre todo por ser las mujeres que migran más jóvenes que los hombres que las acompañan ${ }^{18}$. La combinación de todo este conjunto de factores determinará la estructura por sexo y edad de una población, dando su forma característica a la cúspide de la pirámide.

La pirámide de población del distrito Centro (Fig. 1), como puede fácilmente adivinarse después de haber analizado su estructura por edades y la gravedad de sus índices de envejecimiento, está sumamente distorsionada y se distorsiona aún más con el paso del tiempo. Si la proporción de mujeres que arroja los datos del Censo de 1991 es ya algo más elevada en Madrid (51,8\% frente a $51,0 \%$ en España), las cifras se disparan al detenernos en nuestro ámbito de estudio.

El distrito Centro alcanzaba en 1970 unos índices de población femenina del 55,9\%, mientras los varones suponían el restante $44,1 \%$ (lo que se traducía en una relación de 78,6 hombres por cada 100 mujeres), pero la ratio de masculinidad no disminuye con el paso del tiempo, como sería previsible, al aumentar los índices de envejecimiento. En 1991, las mujeres reducían su representación hasta un 55,6\% (frente al 44,3\% de población masculina), es decir, por cada 100 mujeres residiendo en el distrito habría 79,7 hombres. Por barrios, las ratios de masculinidad y feminidad atestiguan la distorsión que experimenta la composición por edades, especialmente en aquellos situados en la mitad norte del distrito donde paradójicamente las presiones del sector terciario han sido más fuertes, nos estamos refiriendo, como no, a los barrios de Justicia, Universidad, Cortes y Sol (con proporciones de población femenina, en 1970 , que se elevaban al 57,7\%, 56,8\%, 56,1\% y 55,8\%; descendiendo ligeramente en 1991 hasta el 55,7\%, 56\%, 45,4\% y $56,3 \%$ respectivamente), tanto Palacio como Embajadores, a pesar de caracterizarse por estructuras de población claramente feminizadas, se mantienen en tasas inferiores a la media del distrito en ambos recuentos censales $(55,4 \%$ y $54,9 \%$ en 1970 y $55,3 \%$ y $55,5 \%$ en 1991$)$. Estos datos no hacen sino reafirmar las conclusiones obtenidas por el equipo de redacción del PAI-Centro en 1979: las zonas donde es mayor la sustitución de usos residenciales también presentan los mayores porcentajes de po-

18 Este factor explicaría la diferencia que se aprecia entre la Comunidad de Madrid y el conjunto de España. La diferencia se produce sobre todo a partir de los 25 años y, aunque se mantiene hasta el final, es más relevante en el grupo de 25 a 50 años, en el que proliferan los inmigrantes que llegaron entre 1960 y 1975. 
blación en edad avanzada (en este caso reflejada a través de la feminización de la estructura demográfica y no mediante los índices generales de población anciana). En términos generales, y a riesgo de ser reiterativos, hay menos desequilibrios de estructura demográfica el sur del distrito, con la excepción del núcleo interior de Malasaña y algunas zonas de borde donde la renovación ha sido más intensa.

Pero volvamos sobre el problema de la reducción del porcentaje de féminas entre 1970 y 1991, cuando de hecho las tasas de envejecimiento aumentan de forma imparable en el mismo período. Las causas parecen estar vinculadas en este punto al incremento de efectivos masculinos en las cohortes de edad adulta, ya que, tomando el mismo intervalo temporal de referencia, el peso de las mujeres no hace sino incrementarse a medida que aumenta la edad de las mismas. El resultado de este fenómeno es un reequilibrio de la estructura de la población por sexos entre los 25 y los 64 años, situación que desaparece a medida que nos acercamos a los segmentos más envejecidos.

Si la tesis expuesta puede parecer algo confusa, la situación se clarifica al considerar la relación de masculinidad de la población del distrito diferenciándola por grupos de edad, entre 1970 y 1991. Hasta los 15 años el reparto de población entre ambos sexos sigue la lógica marcada por el mayor número de nacimientos de varones que de mujeres, lo que beneficia a los primeros con un porcentaje del $51,2 \%$ frente al $48,8 \%$ de las segundas, relación que se mantiene inamovible desde 1970. En este mismo año, el predominio del sexo masculino se había invertido entre los 15 y los 34 años cuando el peso de los hombres ya había descendido hasta un 49,8\%, superándolos las mujeres con un 50,2\%. Sin embargo, en 1991, las diferencias a favor de los efectivos masculinos conseguirán mantenerse hasta los 34 años de edad, algo más de lo que suele ser habitual, puesto que para el conjunto de la Comunidad de Madrid la inversión se produce a partir de los 25 años, si bien ya con un peso relativo mucho más ajustado (50,5\% de varones y $49,5 \%$ de féminas).

A partir de los 35 años y hasta los 64, la balanza se inclina de forma progresiva hacia el colectivo femenino, pero con intensidades muy diferentes, pues mientras en 1970 los porcentajes de mujeres y hombres ascendían al 58,7\% y 41,3\% respectivamente, en 1991, las primeras rebajan su representación a un 56,6\% del total de residentes, suponiendo los varones el 45,4\% de la población. Pero, en cualquier caso, el distri- 
to Centro esta lejos de alcanzar en este grupo el punto más alto en la distorsión de su estructura por sexos, antes bien, como ya hemos dicho previamente ha conseguido un cierto reequilibrio en su composición interna.

Será entre los 65 y los 74 años cuando este tímido proceso de reestructuración se detenga, en 1970 las mujeres situadas en este grupo acaparaban el 63,4\% del total de residentes frente a un 36,6\% de hombres; los resultados del Censo de 1991 muestran el agravamiento de la situación, ahora ellas representan el 64,4\%, mientras ellos sólo suponen un modesto 35,6\%, es decir, por cada 100 mujeres sólo residen en el distrito 55,4 hombres.

Los desequilibrios parecen haberse estancado al llegar a las cohortes de edad que superan los 75 años, pues si este grupo supone el techo en la distorsión de la pirámide, al menos la misma no se ha agudizado desde nuestro primer año de referencia. Las mujeres, que se distinguían en 1970 con un $71,2 \%$ frente al escaso $28,8 \%$ de los varones, moderan su peso en 1991 con un 70,7\% opuesto al $29,3 \%$ que representan estos últimos, en otras palabras 41,2 hombres por cada 100 mujeres residiendo en el Casco Antiguo.

En resumen, las tasas generales de feminización del distrito, si bien han descendido de forma significativa en los pasados veinte años, continúan reflejando la distorsión que provoca el acusado envejecimiento de sus residentes. El problema se agrava si consideramos que dicho envejecimiento continúa progresando, pues a pesar del aumento de efectivos, especialmente masculinos, que registran las cohortes de población adulta, la inyección que los mismos suponen no ha podido frenar hasta ahora el sobrepeso relativo de la tercera edad. Las consecuencias económicas de esta situación no escapan a nadie familiarizado con el problema, por cuanto en su mayoría este colectivo esta formado por mujeres, en un buen número de casos viudas, que sobreviven con la escasa cuantía de sus pensiones ${ }^{19}$.

${ }^{19}$ La acuciante situación en que sobrevive gran parte del colectivo femenino más envejecido ha sido puesta de relieve por multitud de estudios, y por el propio movimiento ciudadano, que no ha dejado de proponer algunas medidas al respecto. Entre estas últimas, destacan las peticiones que desde la Confederación de Federaciones y Asociaciones de Viudas «Hispania» se han elevado a la Administración a fin de paliar la difícil situación económica que padece este grupo, y que aquí hemos querido destacar por la relevancia que este último tiene entre la población femenina del distrito.

$$
-689-
$$




\section{BIBLIOGRAFÍA}

AA.VV. (1994): V lnforme sociológico sobre la situación social en España Sociedad para todos en el año 2000, Madrid, Fundación FOESSA, 2 vols.

ABELlán GARCÍA, A. (1990): El envejecimiento de la población, tendencias e implicaciones, I.E.G.A., Departamento de Geografía Humana y Regional, C.S.I.C., Documento de Trabajo núm. 1, 86 pp.

Aguilera ArILLA, M. J. y GutiérRez YANCI, P. (1989): «Los contrastes del envejecimiento demográfico entre el municipio de Madrid y su Comunidad Autónoma», II Jornadas sobre la población española, Palma de Mallorca, pp. 67-79.

ARmengaud, A. (1967): «Methods for describing the age-sex structure of cities» (in) GIBBS, J. P. (Ed.): Urban Research Methods, D. Van Nostrand Company, Princenton N.J., $130 \mathrm{pp}$.

BERG, L. van den et al. (eds.) (1982): Urban Europe: a study of growth and decline, Oxford, Pergamon.

BRUNET, R. et al. (1989): Les Villes «Européennes»; Rapport pour la DATAR, Paris, La Documentation Française.

CheshiRe, P. C. y HAY, D. G. (1989): Urban problems in Western Europe: an economic analysis, London, Unwin Hyman.

(1990): «Explaining the recent performance of the European Community's major urban regions», Urban Studies, 2\%, pp. 311-333.

Clark, M. (1971): «Patterns of aging among the elderly poor in the inner city», The Gerontologist, vol. 2, pp. 58-66.

CoLLot, C. (1978): «Les personnes âgées face aux opérations de réhabilitation, restauration, rénovation immobilières», Années Documents CLEIRPPA, 69, janvier, pp. 9-12.

Cotorruelo, R. R..; Olano Rey, A. y AGÜERo Menéndez, I. (1988): Cambios de población en el territorio, MOPU y ITU, Madrid.

Delgado, M. y FernándeZ CoRdón, J. A. (1989): La fecundidad en España desde 1975, Madrid, Instituto de Demografía-CSIC.

FERNÁNDEZ CORDÓN, J. A. (1993): La población y la fecundidad de la Comunidad de Madrid, Informe monográfico del Tomo I de los Censos de Población y Vivienda de 1991 Estudios y Análisis, Departamento de Estadística, Consejería de Economía, Comunidad Autónoma de Madrid.

FOESSA, Fundación (1972): Informe sobre la estructura social de la provincia de Madrid, Fundación FOESSA, Madrid, Euroamérica, 256 pp.

FREY, W. H. (1988): «Migration and metropolitan decline in developed countries. a comparative study», Population and Development Review, vol. 14, 4.

_ (1988): «Migración y despoblamiento de las metrópolis: ¿reestructuración regional o renacimiento rural?», Estudios Territoriales, 28.

FRIEDRICHS, J. (1993): «A theory of urban decline: economy, demography and political elites», Urban Studies, University of Glasgow, 30, 6, pp. 907-917.

GarCÍA BALLESTERos, A. (1977): «Notas sobre el crecimiento natural y real de los distritos de Madrid», Revista Internacional de Sociología, 23.

— _ Pozo Rivera, E.; Crespo Valero, M. J. y ARranz Lozano, M. (1989): «El envejecimiento actual de la población madrileña: diferencias espaciales», II Jornadas sobre la población española, Palma de Mallorca, pp. 207-228.

GarCía Escalona, E. (1990): «Emigración y dinámica urbana en Madrid» Jornadas Internacionales sobre Demografía Urbana y Regional, Madrid, $12 \mathrm{pp}$.

GARRIDO, L. (1993): Las dos biografías de la mujer en España, Madrid, Instituto de la Mujer, Ministerio de Asuntos Sociales.

HALL, P. (1993): «Forces shaping urban Europe», Urban Studies, University of Glasgow, 30,6 , pp. 883-898. 
HERBIN, J. (1987): «Analyse comparative du vieillissement urbain: l'exemple de la région Rhône-Alpes», Espace, Population, Sociétés, 1, pp. 195-206.

HugOnOT, R. et al. (1988): Atlas de la vieillesse et du vieillissement, Ed. Eres, Paris, 456 pp.

Jouvenel, H. de (1989): «Le vieillissement démographique en Europe», Futuribles, 129130 , pp. 53-113.

LAMOURÈRE, O. (1988): Los que vivimos solos. La soledad ya no es lo que era, Paidós, Barcelona.

Le Bras, H. y Chesnais, J. C. (1976): «Cycle de l'habitat et âge des habitants», Population, 2.

LESTHAEGE, R. (1992): «The second demographic transition in western countries: an interpretation", ponencia presentada al Seminar on Gender and Family Change in Industrialized Countries, Roma, IUSSP Comittee on Gender and Population.

LIZANa RIVAS, E. (1985): Distribución y comportamientos espaciales de la población envejecida en Madrid, Tesina presentada en la Universidad Complutense de Madrid, Departamento de Geografía Humana, Madrid, $174 \mathrm{pp}$.

LÓPEz GÓMez, A. (1985): La población de Madrid: del crecimiento a la disminución (1970-1980), Madrid, Real Academia de Doctores de Madrid, 50 pp.

LÓPEZ JimÉnez, J. J. (1993): El envejecimiento y las personas ancianas en Madrid. Area de Servicios Sociales, Ayuntamiento de Madrid, 358 pp.

MERLIN, P. (1986): «Housing politics in the old centre and the development of ghettos of marginal groups» (en) HEINRITZ, G. y LICHTENBERGER, E. (eds.): The take-off of suburbia and the decline of the central city, Stuttgart, Steiner Verlag, pp. 228-234.

MiNISTERIO DE ECONOMÍA Y HACIENDA (1991): Envejecimiento, edad y empleo en Europa, Madrid, Instituto de Estudios de Prospectiva, LXIV, 340 pp.

Ministerio de TRABajo (1990): De la pirámide al pilar de población. Los cambios en la población y la seguridad social en Europa, Madrid, Informes OIT, 252 pp.

NACIONES UNIDAS (1986): «La situación del envejecimiento en el mundo: estrategias y politices», Departamento Internacional de Asuntos Económicos y Sociales, New York, $302 \mathrm{pp}$.

Newcombe, C. (1961): «Graphic representation of age and sex distribution of population in the city», (In) HATT Y REISS (Ed.): Cities and Society, Ed. Glencoe, Illinois.

Paniagua MazorRa, A. (1989): «Pensiones públicas y pobreza en el Tercera Edad», Documentos de trabajo del Departamento de Geografía Humana y Regional, C.S.I.C., $22 \mathrm{pp}$.

PEACE, S. M. (1982): An international perspective on the status of older women, International Federation on Ageing, Washington, $92 \mathrm{pp}$.

Pearce, D. M. (1983): «The feminization of ghetto poverty», Society, 21, pp. 71-74.

PuYol ANTOLIN, R. (1987): «El uso de los modelos de diferenciación espacial de innovaciones en el estudio geográfico de la fecundidad», Anales de Geografía de la Universidad Complutense, 7, pp. 185-191.

-; Vinuesa ANGUlo, J. y ABELlÁN GARCíA, A. (1993): Los grandes problemas actuales de la población, Madrid, Síntesis, $236 \mathrm{pp}$.

RHein, P. (1987): «Transformation des structures urbaines et vieillissement démographique dans l'agglomération parisienne. 1954-1982», Espace, Population, Sociétés, 1, pp. 153170.

RodríGuez Rodríguez, V. y RoJo PÉRez, F. (1989): «La evolución del envejecimiento en España desde 1900 a 1986; distribución espacial», II Jornadas sobre la Población Española, Palma de Mallorca, mayo, pp. 381-402.

Rowles, G. D. (1986): «The geography of ageing and the aged: toward an integrated perspective», Progress in Human Geography,4, pp. 511-539.

SERRANO TORREGO, F. (1990): «Los movimientos migratorios madrileños en las últimas dé- 
cadas y su incidencia espacial», Jornadas Internacionales sobre Demografía Urbana y Regional, Madrid, $14 \mathrm{pp}$.

VIDAL, T. y RECAÑo, J. (1988): «Consecuencias económicas y sociales de la post-transición demográfica en Europa», Rev. Situación, 3, pp. 49-77.

Vinuesa Angulo, J. (1990): Proceso de envejecimiento de la población de Madrid, I.E.G.A., Departamento de Geografía Humana y Regional, C.S.I.C., Documentos de Trabajo, 8, $38 \mathrm{pp}$

- J. (1991): «Modelo de crecimiento metropolitano y desequilibrios en la estructura demográfica» (en) Gosalvez PÉREz, V. (Coord.): Los procesos de urbanización siglos XIX $y$ XX, Alicante, Instituto de Cultura Juan Gil-Albert, pp. 63-78.

WALKER, A. (1980): «The social creation of poverty and dependency in old age», Journal of Social Policy, 9, pp. 49-75.

- (1986): «Pensions and the production of poverty in the old age», (in) PHILLIPSON, Ch. y Walker, A.: Aging and social Policy, Aldershol, Vermont Gower Publishing, pp. 184216

WILSON, W. J. (1987): The truly disadvantaged: the inner city, the underclass and public policy, Chicago, University of Chicago Press.

WinChester, H. P. M. y WhiTe, P. E. (1988): «The location of marginalized groups in the inner city», Environment and Planning D: Society and Space, 6, pp. 37-54.

YNZENGA, B. (Dir.) (1979): PAI-Centro, vol. 10: «Población: proyecciones y datos complementarios», Madrid, COPLACO, MOPU, estudio no publicado.

ZaMORA LóPEZ, F. et al. (1989): 2011: Proyecciones de la población de la Comunidad de Madrid, Ayuntamiento de Madrid: Departamento de Estudios y Análisis, Comunidad de Madrid: Consejería de Economía, C.S.I.C.: Instituto de Demografía, 160 pp.

-; SerRano Secanella, P. y Fernández Acebes, A. I. (1993): Proyecciones de poblaciones y hogares de la Comunidad de Madrid, 1991-2006. Informe 3. Hipótesis de evolución futura de los componentes demográficos de la Comunidad de Madrid, 19912006. Resultados de las proyecciones cerradas, Serie Informes, Instituto de Demografía, C.S.I.C., Madrid. 